\title{
Molecular Basis for Enhancement of the Meiotic DMC1 Recombinase by RAD51AP1
}

Eloïse Dray ${ }^{\mathrm{a}, 1,2}$, Myun Hwa Dunlop ${ }^{\mathrm{a}, 1}$, Liisa Kauppi ${ }^{\mathrm{b}}$, Joseph San Filippo ${ }^{\mathrm{a}, \mathrm{c}}$, Claudia Wiese $^{\mathrm{d}}$, Miaw-Sheue Tsai ${ }^{\mathrm{d}}$, Sead Begovic ${ }^{\mathrm{a}}$, David Schild ${ }^{\mathrm{d}}$, Maria Jasin ${ }^{\mathrm{b}, \mathrm{c}}$, Scott Keeney $^{\text {bee }}$,Patrick Sung ${ }^{\mathrm{a}, 2}$.

a Department of Molecular Biophysics and Biochemistry, Yale University School of Medicine, New Haven, CT 06520, USA

${ }^{\mathrm{b}}$ Molecular Biology Program, Memorial Sloan-Kettering Cancer Center, New York, NY, USA

'Present address: Roche Molecular Systems, Inc., 4300 Hacienda Drive, Pleasanton, CA 94588

${ }^{\mathrm{d}}$ Life Sciences Division, Lawrence Berkeley National Laboratory, Berkeley, CA 94720, USA

${ }^{e}$ Howard Hughes Medical Institute, Memorial Sloan-Kettering Cancer Center, New York, NY, USA

\begin{abstract}
Homologous recombination is needed for meiotic chromosome segregation, genome maintenance, and tumor suppression. RAD51AP1 (RAD51 Associated Protein 1) has been shown to interact with and enhance the recombinase activity of RAD51. Accordingly, genetic ablation of RAD51AP1 leads to enhanced sensitivity to and also chromosome aberrations upon DNA damage, demonstrating a role for RAD51AP1 in mitotic homologous recombination. Here we show physical association of RAD51AP1 with the meiosis-specific recombinase DMC1 and a stimulatory effect of RAD51AP1 on the DMC1-mediated D-loop reaction. Mechanistic studies have revealed that RAD51AP1 enhances the ability of the DMC1 presynaptic filament to capture the duplex DNA partner and to assemble the synaptic complex, in which the recombining DNA strands are homologously aligned. We also provide evidence that functional co-operation is dependent on complex formation between DMC1 and RAD51AP1, and that distinct epitopes in RAD51AP1 mediate interactions with RAD51 and DMC1. Finally, we show that RAD51AP1 is expressed in mouse testes, and that RAD51AP1 foci co-localize with a subset of DMC1 foci in spermatocytes. These results suggest that RAD51AP1 also serves an important role in meiotic homologous recombination.
\end{abstract}

Vbody 


\section{Introduction}

In both prokaryotes and eukaryotes, homologous recombination represents an important pathway for the repair of DNA double-strand breaks (DSBs) and other deleterious chromosomal lesions (1). Defects in HR lead to destabilization of the genome and represent a progenitor of the tumor phenotype in mammals. Moreover, HR in meiosis generates crossovers between homologous chromosomes that are essential for accurate chromosome segregation during the first meiotic division (1-3). Accordingly, HR mutants often exhibit severe meiotic phenotypes, including prophase arrest or apoptosis (4). In meiotic cells, genome-wide HR events, triggered via the introduction of programmed DSBs into chromosomes by the Spo11 complex, are often channeled into the interhomolog HR pathway to be resolved as crossovers $(5,6)$.

The eukaryotic HR reaction is mediated by RAD51 or DMC1, recombinases that are orthologous to the bacterial RecA protein (7-9). Like RecA, RAD51 and DMC1 function within the context of a right-handed helical polymer assembled on ssDNA $(10,11)$. This recombinase-ssDNA nucleoprotein filament, commonly referred to as the presynaptic filament, undergoes a conformational change upon ATP-binding such that it becomes activated for homologous DNA pairing and strand exchange $(12,13)$. Furthermore, the presynaptic filament possesses a second binding site that can accommodate a duplex DNA molecule $(14,15)$. As such, the presynaptic filament provides the catalytic scaffold to sample the incoming duplex DNA and form first an unstable paranemic joint with the ssDNA, which can mature into a stable plectonemic joint in which the recombining DNA strands are linked topologically (16-19).

A variety of recombinase partner proteins that have specific attributes germane for the enhancement of the HR reaction have been identified $(18,19)$. RAD51AP1 (RAD51 Associated Protein 1), so named because of its ability to associate with human RAD51 in the yeast two-hybrid assay (20), is a vertebrate-specific protein that interacts with RAD51 directly and enhances the ability of RAD51 to mediate the D-loop reaction $(21,22)$. RAD51AP1 knockdown by RNA interference leads to cellular sensitivity to DNA damaging agents and elevated DNA damage-induced chromatid breaks $(21,22)$. Enhancement of the RAD51 recombinase activity by RAD51AP1 is contingent upon physical interaction of the two HR factors $(21,22)$. It is not clear whether RAD51AP1 is involved in meiotic recombination, and, if so, whether it functions with RAD51, DMC1, or both recombinases.

Here, we document studies that reveal physical interaction of RAD51AP1 with the meiotic recombinase DMC1 and strong enhancement of the DMC1-mediated D-loop reaction by RAD51AP1 in vitro. We provide evidence that RAD51AP1 functions in conjunction with the DMC1 presynaptic filament to capture the duplex DNA partner and to assemble the synaptic complex, in which the recombining DNA molecules are joined paranemically. Moreover, using spliced forms and mutant variants of RAD51AP1, we show that physical interaction of the RAD51AP1-DMC1 pair is indispensable for their functional synergy. We present evidence that RAD51AP1 is abundantly expressed in 
mouse meiosis and that RAD51AP1 co-localizes with DMC1 in foci on meiotic chromatin. Our findings provide insights into the mechanistic role that RAD51AP1 fulfills in HR reactions and suggest that RAD51AP1 is also a regulator of meiotic HR.

\section{Results}

Purification of RAD51AP1 Isoforms. RAD51AP1 that is soluble and functionally active can be expressed in $E$. coli in various tagged forms (22). Three RAD51AP1 isoforms that harbor 352 residues (Isoform 1), 335 residues (Isoform 2), or 302 residues (Isoform 3) were used in the present work (Fig. 1A). Although all three isoforms were found to interact with RAD51 (20-22), Isoform 2 was used for initial studies with DMC1 as it is the dominant form in humans and is the only documented isoform in some species (Table S1). Multi-step procedures that encompass affinity and conventional chromatographic steps have been devised (22) (Fig. 1B) to purify the above-mentioned RAD51AP1 isoforms to near homogeneity (Fig. 1C).

Complex of RAD51AP1 and DMC1. An in vitro affinity pulldown assay that made use of glutathione Sepharose was employed to examine the ability of GST-tagged RAD51AP1 Isoform 2 to interact with human DMC1. As shown in Fig. 1D, a RAD51AP1-DMC1 complex could be readily captured. This complex is specific, as little if any of $S$. pombe Dmc1 (SpDmc1) associated with RAD51AP1 under the same conditions (Fig. 1D). As reported before (22), neither S. cerevisiae Rad51 (yRad51) nor $E$. coli RecA could form a complex with RAD51AP1. We repeated the affinity pulldown experiments with MBP-tagged RAD51AP1 Isoform 2 and found that the tag has no effect on DMC1 interaction (see below). Taken together, these results revealed that RAD51AP1 physically interacts with DMC1.

Enhancement of DMC1-mediated D-loop Formation by RAD51AP1. In light of the newly discovered interaction between RAD51AP1 and DMC1, we questioned whether RAD51AP1 would enhance the DMC1-mediated D-loop reaction. Indeed, while DMC1 alone converted only $\sim 3 \%$ of the input oligonucleotide into the D-loop product (Fig. $1 E$ ), up to 8-fold increase of product formation occurred when RAD51AP1 Isoform 2 was added (Fig. 1E). RAD51AP1 alone is devoid of D-loop forming ability $(21,22)$ (see later), and its stimulatory effect on DMC1 is specific, as no such enhancement was observed when SpDmc1 was used instead (Fig. 1E). As expected, the D-loop reaction mediated by the combination of DMC1 and RAD51AP1 is strictly ATP dependent (Fig. $1 E$ ). Taken together, the results revealed an ability of RAD51AP1 to up-regulate the recombinase activity of DMC1.

The above D-loop experiments were conducted with $\mathrm{Mg}^{2+}$ ion wherein turnover of the recombinase from ssDNA occurs upon ATP hydrolysis. Importantly, RAD51AP1 also stimulated D-loop formation when $\mathrm{Mg}^{2+}$ was substituted by $\mathrm{Ca}^{2+}$ (Fig. S1A) to stabilize the DMC1 presynaptic filament (23). These results, coupled with the fact that RAD51AP1 binds dsDNA preferentially over ssDNA (22), indicated that RAD51AP1, in its role as an enhancer of the DMC1 recombinase function, likely acts at a step 
subsequent to the assembly of the presynaptic filament, a premise that will be elaborated upon below.

Promotion of Duplex Capture and Synaptic Complex Assembly by RAD51AP1. During the homologous pairing process that results in D-loop formation, the presynaptic filament first engages the dsDNA partner and then conducts a search to locate homology in the partner. This leads to the assembly of a nucleoprotein ensemble termed the synaptic complex, in which the recombining ssDNA and dsDNA are homologously aligned and stabilized by protein and DNA interactions $(16,24)$. We queried whether RAD51AP1 functions with the DMC1 presynaptic filament to engage duplex DNA and promote synaptic complex assembly.

We first employed an assay, devised by us previously (25), to test the effect of RAD51AP1 on duplex-DNA capture. For this purpose, the DMC1 presynaptic filament was assembled on ssDNA linked to magnetic beads via a streptavidin-biotin bridge. Then, the ability of the presynaptic filament to capture a radiolabeled duplex DNA of unrelated sequence to the bound ssDNA was examined, either in the absence or presence of RAD51AP1 (see Fig. 2A). In agreement with previously published results (26), the DMC1 presynaptic filament on its own had only a limited ability to capture duplex DNA (Fig. 2B). Importantly, even though RAD51AP1 by itself did not capture the duplex (Fig. $2 B)$, its incubation with the DMC1 presynaptic filament led to a strong enhancement of duplex capture (Fig. 2B). The duplex capture reaction showed a strict dependence on ATP (Fig. 2B), indicating that a functional DMC1 presynaptic filament was needed. Interestingly, the combination of the DMC1 presynaptic filament and RAD51AP1 was much less adept at capturing ssDNA (Fig. 2C), consistent with the dsDNA binding preference of RAD51AP1 (22). As in the D-loop reaction (Fig. 1E), enhancement of duplex capture by RAD51AP1 is specific to human DMC1, as no such effect was seen with SpDmc1 (Fig. S2A). In addition, the DMC1 presynaptic filament stabilized by $\mathrm{Ca}^{2+}$ ion (23) was also unable to capture duplex DNA unless RAD51AP1 was included (Fig. $\mathrm{S} 1 C$ ). As expected, duplex capture was contingent upon the presence of ssDNA on the magnetic beads (Fig. S1B).

We next assessed synaptic complex formation $(26,27)$ by DMC1 and RAD51AP1. This assay involves monitoring the protection of linear dsDNA by a DMC1 presynaptic filament harboring homologous ssDNA against digestion by the restriction enzyme SspI (Fig. 2D). As expected from published work (26), the DMC1 presynaptic filament alone was poorly adept at assembling the synaptic complex (Fig. 2E). Importantly, the addition of RAD51AP1 afforded a marked stimulation of synaptic complex assembly, in a manner that required ATP (Fig. 2E). Control reactions revealed that RAD51AP1 alone is not able to assemble the synaptic complex (Fig. $2 E$ ) nor does it synergize with the SpDmc1 presynaptic filament in the reaction (Fig. S2B).

Requirement for DMC1-RAD51AP1 Interaction in Synaptic Complex Assembly. We verified that RAD51AP1 Isoform 1, previously used in the study of Modesti et al (2007), is just as capable as RAD51AP1 Isoform 2 in DMC1 interaction (Fig. 3A) and in enhancement of the D-loop reaction (Fig. 3B). Interestingly, we found that RAD51AP1 
Isoform 3, which lacks a segment of the C-terminus of the other two isoforms (Fig. 1A), while being capable of RAD51 interaction (Fig. S3A), is deficient in DMC1 interaction (Fig. 3A). Accordingly, results from D-loop reactions revealed that while RAD51AP1 Isoform 3 is just as active as the other two isoforms in enhancing RAD51's recombinase activity (Fig. S3B) and in DNA binding (Fig. S4), it is defective in the promotion of the DMC1-mediated D-loop reaction (Fig. 3B).

Subsequently, we wanted to test whether duplex DNA capture and synaptic complex assembly would likewise be dependent on RAD51AP1-DMC1 interaction. Indeed, while RAD51AP1 Isoform 1 was just as capable as RAD51AP1 Isoform 2 in the promotion of duplex capture and synaptic complex assembly, RAD51AP1 Isoform 3 proved to be inactive in these regards (Fig. $3 C$ and $3 D$ ).

RAD51AP1 Mutants Defective in RAD51 Association are Proficient in DMC1 Interactions. Previous studies isolated two point mutants of RAD51AP1 Isoform 2, namely, L319Q and H329A (28), that are both deficient in physical interaction with RAD51 and in enhancement of the RAD51-mediated D-loop reaction (22). Interestingly, these RAD51AP1 mutant proteins (Fig. S5A) were just as proficient as the wild type counterpart in DMC1 interaction (Fig. S5B). When tested in the D-loop, duplex capture, and synaptic complex assembly assays, the two mutants showed a degree of stimulatory activity comparable to that of RAD51AP1 Isoform 1 or Isoform 2 (Fig. S5, C-E). Overall, these results revealed that RAD51AP1 residues that are critical for RAD51 interaction are dispensable for physical association and functional synergy with DMC1.

Meiotic Expression of RAD51AP1. We conducted reverse transcriptase (RT)-PCR with total RNA isolated from testes of juvenile mice $(5,15$, and 21 days post partum (dpp); Fig. S6B), with the three different age samples being enriched for premeiotic, midprophase I, and late prophase I meiotic cells (29). Even though three RAD51AP1 isoforms exist in human cells, only one isoform of RAD51AP1 has been documented in mice (Table S1; 30) and this is equivalent to human RAD51AP1 Isoform 2 that possesses the exon needed for DMC1 interactions (Fig. S6A). RT-PCR and immunoblot analyses revealed that mouse RAD51AP1 is abundantly expressed in 15 and $21 \mathrm{dpp}$ testes (Fig. $\mathrm{S} 6, B-C)$. We note that our results are in concordance with previous reports $(28,30)$ showing presence of the RAD51AP1 transcript in human or mouse testes.

Co-localization of RAD51AP1 with DMC1 on Meiotic Chromatin. Next we wanted to ask if RAD51AP1 co-localizes with DMC1 in meiotic cells. DMC1 appears as discrete foci on meiotic chromatin during the leptotene and zygotene stages (4, 31). Meiotic chromosome spreads were prepared from spermatocytes of juvenile mice and triplestained with antibodies raised against SYCP3, DMC1 and RAD51AP1. SYCP3 is a component of meiotic chromosome axes $(32,33)$ used to monitor meiotic progression (34). Axes of homologous chromosomes lengthen during leptotene stage and gradually pair and become intimately juxtaposed at zygotene to pachytene stages of meiotic prophase I to form the synaptonemal complex. 
Several classes of RAD51AP1 foci could be discerned (Fig. S7, Fig. S9, and companion description online); below, we focus on axis-associated Class I foci. Among 16 leptotene spermatocytes analyzed (Fig. 4A), frequent co-localization of RAD51AP1 with DMC1 was observed (Fig. $4, B-D$ ). These nuclei had $135 \pm 73 \mathrm{DMC} 1$ foci and $129 \pm 79$ Class I RAD51AP1 foci, of which $51 \pm 37$ foci stained with antibodies to both proteins (Fig. $4 G)$. The fraction of DMC1 foci positive for RAD51AP1 was $38 \pm 17 \%$, and the fraction of RAD51AP1 foci positive for DMC1 was $42 \pm 23 \%$ (Fig. $4 H$ ). The specificity of the DMC1 and RAD51AP1 antibodies used was established by treatment with an excess of the cognate protein to deplete the available antibodies (Fig. S9).

Since the nuclear localization of RAD51AP1 appears complex (Fig. S7), we tested whether any or all of the co-localization of RAD51AP1 with either SYCP3-stained axes or DMC1 foci could have arisen from fortuitous spatial proximity of unrelated protein distributions $(35,36)$. First, we counted co-localizing RAD51AP1 and DMC1 foci within $20 \mu \mathrm{m} \times 20 \mu \mathrm{m}$ boxes of leptotene nuclei (Fig. $4 A$ and $4 E$ ). We then rotated the RAD51AP1 image within each box by $180^{\circ}$ and re-scored foci that co-localized with axes and DMC1 (Fig. $4 F$ ). Upon rotating the DMC1 image as a control analysis, the count was decreased from $41 \pm 18$ axis-associated DMC1 foci in the true images to $12 \pm$ 4 axis-associated foci in the rotated images, validating this method (Fig. 4I, $P=0.0013$, Mann-Whitney $U$-test). In contrast, the spreads with the rotated RAD51AP1 channel displayed on average $35 \pm 14$ axis-associated RAD51AP1 foci, which is statistically indistinguishable from the $30 \pm 8$ observed in the unrotated nuclei (Fig. $4 I, P=0.3696$ ). Thus, the association of RAD51AP1 foci with the axes may not always be specific. Importantly, however, when we counted DMC1 and RAD51AP1 co-localized foci before and after $180^{\circ}$ rotation of the RAD51AP1 image, the number of co-localized DMC1 decreased from $21 \pm 11$ to $7 \pm 3(P=0.0027)$. This corresponds to a decrease from $48 \pm$ $20 \%$ of DMC1 foci that co-localize with RAD51AP1 foci to $25 \pm 15 \%$ (Fig. $4 J$, $P=0.0043)$. Thus, DMC1 foci co-localize with RAD51AP1 foci much more frequently than expected by chance, which in turn supports the conclusion that at least a subset of DMC1-containing, chromosome-associated HR complexes also contain RAD51AP1.

RAD51AP1 and RAD51 co-localize to DNA damage in mitotic cells $(21,22,30)$. Our analysis of 14 leptotene spermatocytes for the foci pattern of RAD51AP1 with RAD51 in meiotic chromatin spreads found frequent co-localization of RAD51AP1 with RAD51 (Fig. S8, $A-D$ and Fig. S9). Specifically, the boxed areas of these nuclei had $64 \pm 17$ RAD51 foci and $84 \pm 18$ Class I RAD51AP1 foci (Fig. S8I). The fraction of RAD51 foci positive for RAD51AP1 was $49 \pm 17 \%$ (Fig. S8J), a number that is very similar to the fraction of DMC1 foci positive for RAD51AP1. Importantly, upon rotation of the RAD51AP1 image (Fig. S8I, J), the number of co-localized RAD51 foci decreased from $30 \pm 9$ to $7 \pm 2(P=0.0004)$. RAD51AP1 foci therefore co-localized with RAD51 more frequently than expected by chance. We also established the specificity of the antiRAD51 antibody by pre-treating it with purified RAD51 protein (Fig. S9).

Thus, RAD51AP1 foci co-localize frequently with either DMC1 or RAD51, and the RAD51AP1-DMC1 association appears to be as intimate as the RAD51AP1-RAD51 association. Since the antibodies against RAD51 and DMC1 were both raised in rabbits, 
we have been unable to perform double staining of RAD51-DMC1 or triple staining of RAD51-DMC1-RAD51AP1 to ascertain whether RAD51AP1 forms a tripartite complex at DSB sites.

\section{Discussion}

Published studies have provided ample evidence for a role of RAD51AP1 in mitotic HR via enhancement of the recombinase activity of RAD51 (20-22). The molecular basis for this enhancement has not yet been determined, although it has been suggested that through its DNA structure specific binding activity, RAD51AP1 may stabilize the D-loop product made by RAD51 (21). We have shown in this study that RAD51AP1 also physically interacts with and stimulates the recombinase activity of DMC1. Using a variety of biochemical tools, we have provided evidence that RAD51AP1 synergizes with the DMC1 presynaptic filament to capture duplex DNA and assemble the synaptic complex. These attributes of RAD51AP1 are likely dependent on its duplex DNA binding activity (22). Importantly, using naturally occurring isoforms of RAD51AP1 and mutant variants of one of these isoforms, we have furnished evidence that the functional interaction of RAD51AP1 with DMC1 is dependent on complex formation between the two HR factors and that distinct epitopes in RAD51AP1 mediate its respective interactions with DMC1 and RAD51. Interestingly, a single RAD51AP1 isoform that is most similar to human Isoform 2 is expressed in the mouse testis. Importantly, immunofluorescence studies of meiotic chromatin spreads showed co-localization of RAD51AP1 with DMC1 in leptonema. Even though we have presented biochemical results and cytological evidence to suggest that RAD51AP1 physically and functionally interacts with $\mathrm{DMC1}$, in vivo studies entailing animal models will be needed to determine if these interactions are biologically meaningful.

Despite the critical role of DMC1 in the formation of meiotic chromosome crossovers needed for the proper disjunction of homologous chromosomes in meiosis I, much remains to be learned about its functional partners (6). The HOP2-MND1 complex is one such DMC1 partner. Biochemical studies have shown that, like RAD51AP1 $(21,22)$, HOP2-MND1 preferentially binds dsDNA over ssDNA $(25,26)$ and promotes DMC1mediated D-loop formation via functional synergy with the DMC1 presynaptic filament in duplex capture and synaptic complex assembly (26). We have found no evidence for physical interaction of RAD51AP1 and HOP2-MND1 or functional synergy of these two factors in the enhancement of the DMC1-mediated D-loop reaction. Moreover, an excess of HOP2-MND1 was unable to prevent the formation of the RAD51AP1-DMC1 complex. We note that cytological analyses done in $S$. cerevisiae and $A$. thaliana have shown that MND1 localizes to chromatin as foci independently of Spo11-mediated DNA double-strand break formation (37-39). Curiously, in S. cerevisiae and A. thaliana, Hop2Mnd1 does not seem to co-localize significantly with Rad51 or Dmc1, implying a transient nature of their interaction or that only a small fraction of Hop2-Mnd1 is available for the enhancement of Rad51 and Dmc1 $(38,39)$. In addition to functioning as a co-factor of DMC1, HOP2-MND1 could also fulfill other roles in meiotic chromosome metabolism. The precise relationship between RAD51AP1 and HOP2-MND1, for instance, whether they provide the same recombinase enhancement function but in distinct sites of the meiotic chromatin, remains to be determined. 


\section{Materials and Methods}

Purification of HR Proteins. GST- and MBP-tagged RAD51AP1 isoforms and mutants were expressed in E. coli and purified by a multi-step procedure, as summarized in Fig. 1B. Human DMC1 (10), SpDmc1 (40), and ScRad54 (41) were purified as described previously.

Affinity Pulldown. The indicated GST-tagged or MBP-tagged RAD51AP1 isoform (5 $\mu \mathrm{g}$ ) was incubated with either (His) ${ }_{6}$-DMC1 or SpDmc1 (5 $\mu \mathrm{g}$ each) in $30 \mu 1$ of buffer $\mathrm{P}$ (25 mM Tris- $\mathrm{HCl}, \mathrm{pH} 7.5,60 \mathrm{mM} \mathrm{KCl}, 1 \mathrm{mM}$ 2-mercaptoethanol) for $30 \mathrm{~min}$ at $4^{\circ} \mathrm{C}$. After being mixed with $7 \mu \mathrm{l}$ of glutathione resin (Amersham) or amylose resin (New England Biolabs) for $30 \mathrm{~min}$ at $4^{\circ} \mathrm{C}$ to capture the tagged RAD51AP1 and associated proteins, the beads were washed three times with $30 \mu \mathrm{l}$ of the same buffer and then treated with $20 \mu \mathrm{l}$ of $2 \%$ SDS to elute proteins. The supernatant, last wash, and SDS eluate, $10 \mu \mathrm{l}$ each, were analyzed by SDS-PAGE.

D-loop Assay. The D-loop assay was conducted at $37^{\circ} \mathrm{C}$, as described $(22,25)$. Briefly, the ${ }^{32} \mathrm{P}$-labeled 90 -mer oligonucleotide substrate $(2.4 \mu \mathrm{M}$ nucleotides $)$ was incubated for 5 min with DMC1 or SpDmc1 $(0.8 \mu \mathrm{M})$ in $10.5 \mu 1$ buffer D (35 mM Tris-HCl, pH 7.5, 50 $\mathrm{mM} \mathrm{KCl}, 2 \mathrm{mM}$ ATP, $4 \mathrm{mM} \mathrm{MgCl}, 100 \mu \mathrm{g} / \mathrm{ml} \mathrm{BSA}$, and $1 \mathrm{mM}$ dithiothreitol), followed by the addition of the indicated amount of RAD51AP1 or ScRad54 in $1 \mu 1$ and a 5-min incubation. pBluescript replicative form I DNA ( $35 \mu \mathrm{M}$ base pairs) was then incorporated in $1 \mu 1$, and after a 20 -min incubation, the reaction mixtures were analyzed $(22,25)$.

Duplex Capture Assay. The assay followed our published procedure (25). To assemble the presynaptic filament, $4 \mu 1$ of magnetic beads containing 5'-biotinylated 83-mer dT ssDNA (60 ng, corresponding to $9 \mu \mathrm{M}$ nucleotides) were incubated with $2.7 \mu \mathrm{M}$ of $\mathrm{DMC} 1$ or SpDmc1 in $18 \mu \mathrm{l}$ of buffer D for $5 \mathrm{~min}$ at $37^{\circ} \mathrm{C}$. The beads were captured with the Magnetic Particle Separator, washed once with $20 \mu \mathrm{l}$ buffer D, and then resuspended in $15 \mu \mathrm{l}$ of the same buffer. Following the incorporation of the indicated amount of RAD51AP1 and a 5-min incubation at $37^{\circ} \mathrm{C}$, the beads were again captured, washed once with $150 \mu 1$ buffer $\mathrm{D}$, and resuspended in $18 \mu \mathrm{l}$ buffer $\mathrm{D}$. The reaction was completed by adding radiolabeled dsDNA ( $4 \mu \mathrm{M}$ base pairs) with or without radiolabeled ssDNA (4 $\mu \mathrm{M}$ nucleotides) in $2 \mu \mathrm{l}$. The reaction mixtures ( $20 \mu \mathrm{l}$ final volume) were incubated for $10 \mathrm{~min}$ at $37^{\circ} \mathrm{C}$ with gentle mixing every $30 \mathrm{sec}$. The beads were captured, washed twice with $200 \mu \mathrm{l}$ of buffer D, and the bound proteins and radiolabeled DNA were eluted with $20 \mu 1$ of $2 \%$ SDS. The supernatant and SDS eluate were resolved in a $10 \%$ polyacrylamide gel in TBE buffer $(90 \mathrm{mM}$ Tris- $\mathrm{HCl}, 70 \mathrm{mM}$ boric acid, $\mathrm{pH} 8.0,2 \mathrm{mM}$ EDTA), and the DNA species were visualized and quantified by phosphorimaging analysis.

Assay for Synaptic Complex Formation. To assemble the presynaptic filament, the 60mer oligonucleotide (26) (12 $\mu \mathrm{M}$ nucleotides) were incubated with $4 \mu \mathrm{M}$ of DMC1 or SpDmc1 in $8 \mu 1$ of buffer D containing $2 \mathrm{mM}$ ATP for $5 \mathrm{~min}$ at $37^{\circ} \mathrm{C}$. The indicated amount of RAD51AP1 isoform or mutant was added in $1 \mu 1$, and, after another 5-min 
incubation at $37^{\circ} \mathrm{C}$, EcoR1-linearized pUC19 DNA (corresponding to $83 \mu \mathrm{M}$ of nucleotides) was added in $1 \mu 1$, followed by the incorporation of 6.5 units of Ssp1 and a further incubation for $10 \mathrm{~min}$ at $37^{\circ} \mathrm{C}$. The reaction mixtures were deproteinized with SDS and proteinase $\mathrm{K}\left(0.1 \%\right.$ and $0.5 \mathrm{mg} / \mathrm{ml}$, respectively) for $5 \mathrm{~min}$ at $37^{\circ} \mathrm{C}$ before being resolved in a $0.9 \%$ agarose gel in TAE buffer $(40 \mathrm{mM}$ Tris, $20 \mathrm{mM} \mathrm{NaOAc}, \mathrm{pH} 7.5,2$ mM EDTA). The DNA species were stained with ethidium bromide and recorded in a BioRad gel documentation station.

Anti-RAD51AP1 Antibodies. MBP-tagged mouse RAD51AP1 was expressed in and purified from $E$. coli by following the procedures employed for MBP-tagged human RAD51AP1 (Fig. 1B). Following cleavage of the MBP tag, the mouse RAD51AP1 protein was used to generate guinea-pig antiserum at Cocalico Biologicals, Inc (PA). This GP49 antiserum was affinity-purified using cyanogen bromide activated Sepharose 4B (Pharmacia-LKB) beads covalently conjugated to the mouse MBP-RAD51AP1 protein, as previously described (41).

Immunofluorescence. Spermatocytes were collected from juvenile (15 days post partum) mouse testes and prepared for surface spreading as previously described (42). Slides were blocked in antibody dilution buffer (1xPBS, $2 \mathrm{mg} / \mathrm{ml} \mathrm{BSA,} 0.05 \%$ Tween-20, $0.2 \%$ gelatin) for $10 \mathrm{~min}$ at $25^{\circ} \mathrm{C}$, and then incubated overnight at $4{ }^{\circ} \mathrm{C}$ with $1: 200$ guinea pig anti-RAD51AP1 antibody (GP49), 1:100 rabbit anti-DMC1 antibody (H-100, Santa Cruz), or 1:650 mouse anti-SYCP3 antibody (D-1, Santa Cruz). Primary antibodies were detected using TexasRed goat anti-mouse IgG, Cy5 goat anti-rabbit IgG or AlexaFluor488 goat anti-guinea pig IgG (Invitrogen). Slides were mounted in Vectashield mounting medium (Vector) with $50 \mu \mathrm{g} / \mathrm{ml}$ 4',5-diamino-2-phenylindole (DAPI, Fluka). Images were captured using an Axio2 microscope (Zeiss) connected to a charge-coupled-device (CCD) camera and processed using the SlideBook software package (Intelligent Imaging Innovations). Leptonema was defined as having short, unsynapsed stretches of SYCP3. Early zygonema was defined as having some $(<30 \%)$ synapsed SYCP3 stretches. Only DMC1 foci that co-localized with SYCP3 staining were counted. The number of colocalizing AP1-DMC1 and AP1-RAD51 foci was scored without restriction to the subset of foci that share the same centroid. Fluorescence channels were aligned by imaging 0.2 $\mu \mathrm{m}$ Tetraspeck beads (Invitrogen). Images were quantified using the Align Channel function in the Slidebook software.

Statistical Analysis of Foci Co-localization. The significance of DMC1 and RAD51AP1 co-localization was tested by performing the Mann-Whitney U-test on the percentage of co-localized foci in the real image versus the rotated image (after RAD51AP1 channel was rotated $180^{\circ}$ ). This test, appropriate to small samples, was chosen as an alternative of the Student test because the two datasets are non-parametric. The DMC1 dataset was drawn from a total of eight spermatocytes $(\mathrm{N}=8)$ and the RAD51 dataset was drawn from a total of nine spermatocytes $(\mathrm{N}=9)$. The Prism 5 software was used to generate two-tail $\mathrm{P}$ values. 


\section{ACKNOWLEDGEMENTS}

SpDmc1 was a kind gift from Peter Chi. We thank Jim Dowdle for helping to prepare IF slides. This work was supported by NIH grants RO1ES015252, RO1HD040916, RO1ES07061, and PO1CA092584, and Susan G. Komen for the Cure Foundation postdoctoral fellowship PDF0706844.

Work at LBNL was under contract no. DE-AC02-05CH11231 from the U.S. Department of Energy.

\section{Footnotes}

Author contributions: E.D., M.H.D., L.K, J.S.F, S.K. and P.S. designed research; E.D., M.H.D., and L.K, performed research and analyzed data; L.K., C.W., M.S.T., S.B., D.S., M.J. and S.K. contributed new reagents/analytic tools; E.D., M.H.D., S.K. and P.S. wrote the paper.

The authors declare no conflict of interest.

${ }^{1}$ E.D. and M.H.D. contributed equally to this work.

${ }^{2}$ To whom correspondence should be addressed. Email: patrick.sung@yale.edu, eloise.dray@yale.edu

This article contains supporting information online.

\section{References}

1. Sung P, Klein H (2006) Mechanism of homologous recombination: mediators and helicases take on regulatory functions. Nat Rev Mol Cell Biol 7:739-750.

2. Symington LS (2002) Role of RAD52 epistasis group genes in homologous recombination and double-strand break repair. Microbiol Mol Biol Rev 66:630-670.

3. Haber JE (1998) Meiosis: Avoiding inappropriate relationships. Curr Biol 8:R832-835.

4. Baudat F, Manova K, Yuen JP, Jasin M, Keeney S (2000) Chromosome synapsis defects and sexually dimorphic meiotic progression in mice lacking Spo11. Mol Cell 6:989-998.

5. Keeney S (2001) Mechanism and control of meiotic recombination initiation. Curr Top Dev Biol 52:1-53.

6. Neale MJ, Keeney S (2006) Clarifying the mechanics of DNA strand exchange in meiotic recombination. Nature 442:153-158.

7. $\quad$ Bishop DK, Park D, Xu L, Kleckner N (1992) DMC1: a meiosis-specific yeast homolog of E. coli recA required for recombination, synaptonemal complex formation, and cell cycle progression. Cell 69:439-456.

8. Shinohara A, Ogawa H, Ogawa T (1992) Rad51 protein involved in repair and recombination in S. cerevisiae is a RecA-like protein. Cell 69:457-470.

9. Shinohara A, et al. (1993) Cloning of human, mouse and fission yeast recombination genes homologous to RAD51 and recA. Nat Genet 4:239-243.

10. Sehorn MG, Sigurdsson S, Bussen W, Unger VM, Sung P (2004) Human meiotic recombinase Dmc1 promotes ATP-dependent homologous DNA strand exchange. Nature 429:433-437. 
11. Sung P, Robberson DL (1995) DNA strand exchange mediated by a RAD51ssDNA nucleoprotein filament with polarity opposite to that of RecA. Cell 82:453-461.

12. Ogawa T, Yu X, Shinohara A, Egelman EH (1993) Similarity of the yeast RAD51 filament to the bacterial RecA filament. Science 259:1896-1899.

13. Yu X, Jacobs SA, West SC, Ogawa T, Egelman EH (2001) Domain structure and dynamics in the helical filaments formed by RecA and Rad51 on DNA. Proc Natl Acad Sci USA 98:8419-8424.

14. Aihara H, Ito Y, Kurumizaka H, Yokoyama S, Shibata T (1999) The N-terminal domain of the human Rad51 protein binds DNA: structure and a DNA binding surface as revealed by NMR. J Mol Biol 290:495-504.

15. Reymer A, Frykholm K, Morimatsu K, Takahashi M, Norden B (2009) Structure of human Rad51 protein filament from molecular modeling and site-specific linear dichroism spectroscopy. Proc Natl Acad Sci USA 106:13248-13253.

16. Bianchi M, DasGupta C, Radding CM (1983) Synapsis and the formation of paranemic joints by E. coli RecA protein. Cell 34:931-939.

17. Gupta RC, Golub E, Bi B, Radding CM (2001) The synaptic activity of HsDmc1, a human recombination protein specific to meiosis. Proc Natl Acad Sci USA 98:84338439 .

18. San Filippo J, Sung P, Klein H (2008) Mechanism of eukaryotic homologous recombination. Annu Rev Biochem 77:229-257.

19. Sung P, Krejci L, Van Komen S, Sehorn MG (2003) Rad51 recombinase and recombination mediators. J Biol Chem 278:42729-42732.

20. Kovalenko OV, Golub EI, Bray-Ward P, Ward DC, Radding CM (1997) A novel nucleic acid-binding protein that interacts with human rad51 recombinase. Nucleic Acids Res 25:4946-4953.

21. Modesti M, et al. (2007) RAD51AP1 is a structure-specific DNA binding protein that stimulates joint molecule formation during RAD51-mediated homologous recombination. Mol Cell 28:468-481.

22. Wiese C, et al. (2007) Promotion of homologous recombination and genomic stability by RAD51AP1 via RAD51 recombinase enhancement. Mol Cell 28:482-490.

23. Bugreev DV, Mazin AV (2004) Ca2+ activates human homologous recombination protein Rad51 by modulating its ATPase activity. Proc Natl Acad Sci USA 101:9988-9993.

24. Riddles PW, Lehman IR (1985) The formation of paranemic and plectonemic joints between DNA molecules by the recA and single-stranded DNA-binding proteins of Escherichia coli. J Biol Chem 260:165-169.

25. Chi P, San Filippo J, Sehorn MG, Petukhova GV, Sung P (2007) Bipartite stimulatory action of the Hop2-Mnd1 complex on the Rad51 recombinase. Genes Dev 21:1747-1757.

26. Pezza RJ, Voloshin ON, Vanevski F, Camerini-Otero RD (2007) Hop2/Mnd1 acts on two critical steps in Dmc1-promoted homologous pairing. Genes Dev 21:1758-1766.

27. Ferrin LJ, Camerini-Otero RD (1991) Selective cleavage of human DNA: RecAassisted restriction endonuclease (RARE) cleavage. Science 254:1494-1497.

28. Kovalenko OV, Wiese C, Schild D (2006) RAD51AP2, a novel vertebrate- and meiotic-specific protein, shares a conserved RAD51-interacting C-terminal domain with RAD51AP1/PIR51. Nucleic Acids Res 34:5081-5092. 
29. Goetz P, Chandley AC, Speed RM (1984) Morphological and temporal sequence of meiotic prophase development at puberty in the male mouse. J Cell Sci 65:249-263.

30. Mizuta R, et al. (1997) RAB22 and RAB163/mouse BRCA2: proteins that specifically interact with the RAD51 protein. Proc Natl Acad Sci USA 94:6927-6932.

31. Tarsounas M, Morita T, Pearlman RE, Moens PB (1999) RAD51 and DMC1 form mixed complexes associated with mouse meiotic chromosome cores and synaptonemal complexes. J Cell Biol 147:207-220.

32. Dobson MJ, Pearlman RE, Karaiskakis A, Spyropoulos B, Moens PB (1994) Synaptonemal complex proteins: occurrence, epitope mapping and chromosome disjunction. J Cell Sci 107(Pt10):2749-2760.

33. Lammers JH, Offenberg HH, van Aalderen M, Vink AC, Dietrich AJ, Heyting C (1994) The gene encoding a major component of the lateral elements of synaptonemal complexes of the rat is related to X-linked lymphocyte-regulated genes. Mol Cell Biol 14:1137-1146.

34. Moens PB, Pearlman RE (1988) Chromatin organization at meiosis. Bioessays 9:151-153.

35. Gasior SL, Wong AK, Kora Y, Shinohara A, Bishop DK (1998) Rad52 associates with RPA and functions with rad55 and rad57 to assemble meiotic recombination complexes. Genes Dev 12:2208-2221.

36. Kee K, Protacio RU, Arora C, Keeney S (2004) Spatial organization and dynamics of the association of Rec102 and Rec104 with meiotic chromosomes. Embo J 23:1815-1824.

37. Tsubouchi H, Roeder GS (2003) The importance of genetic recombination for fidelity of chromosome pairing in meiosis. Dev Cell 5:915-925.

38. Zierhut C, Berlinger M, Rupp C, Shinohara A, Klein F (2004) Mnd1 is required for meiotic interhomolog repair. Curr Biol 14:752-762.

39. Vignard J, et al. (2007) The interplay of RecA-related proteins and the MND1HOP2 complex during meiosis in Arabidopsis thaliana. PLoS Genet 3(10):1894-906.

40. Chi P, et al. (2009) Functional interactions of meiotic recombination factors Rdh54 and Dmc1. DNA repair (Amst) 8:279-284.

41. Sigurdsson S, Van Komen S, Petukhova G, Sung P (2002) Basis for avid homologous DNA strand exchange by human Rad51 and RPA. J Biol Chem 277:4279042794.

42. Barchi M, et al. (2005) Surveillance of different recombination defects in mouse spermatocytes yields distinct responses despite elimination at an identical developmental stage. Mol Cell Biol 25:7203-7215.

\section{FIGURE LEGENDS}

Fig. 1. Characterization of RAD51AP1 isoforms.

(A) The three human RAD51AP1 isoforms used in this study.

(B) Purification procedures for MBP-tagged (I) and GST-tagged (II) RAD51AP1 proteins.

(C) SDS-PAGE analysis of the purified tagged RAD51AP1 isoforms. 
(D) In a pulldown assay, GST-tagged RAD51AP1 Isoform 2 was found to associate with human DMC1 (lane 3) but not with S. pombe Dmc1 (SpDmc1; lane 6). Control reactions were carried out with GST. The supernatant (S), wash (W), and SDS eluate (E) of the pulldown reactions were analyzed by SDS-PAGE.

(E) RAD51AP1 Isoform 2 specifically stimulates D-loop formation by human DMC1. (I) Schematic of the D-loop assay. (II) RAD51AP1 (50, 100, 200, 300 or $500 \mathrm{nM})$ enhanced the D-loop reaction mediated by human DMC1 (lanes 3 to 7) in an ATP-dependent fashion (lane 8). The recombinase activity of SpDmc1 was refractory to RAD51AP1 (200, 300, or $500 \mathrm{nM}$; lanes 9 to 11) but was enhanced by ScRad54 (200 nM; lane 12) in an ATP-dependent fashion (lane 13). (III) The results were plotted; error bars represent the standard deviation $( \pm \mathrm{SD})$ calculated based on at least three independent experiments.

Fig. 2. Functional synergy of RAD51AP1 and DMC1 in duplex capture and synaptic complex assembly.

(A) Schematic of the duplex capture reaction.

(B) DMC1 alone (lane 2) or RAD51AP1 alone (lanes 3 and 4) exhibited little duplex capture activity. DMC1 synergized with RAD51AP1 (300 or $600 \mathrm{nM}$ ) in duplex DNA capture (lanes 5 and 6), with a dependence on ATP (lane 7). The percentages of captured DNA were quantified and plotted in (II). Error bars represent \pm SD calculated based on at least three independent experiments.

(C) When presented with both ssDNA and dsDNA, DMC1 and RAD51AP1 (900 nM) captured the dsDNA preferentially (lane 5). The appropriate controls were included. The results were quantified and plotted (II).

(D) The basis for the protection of the target DNA against digestion by the restriction enzyme SspI is illustrated.

(E) RAD51AP1 $(0.5$ or $1 \mu \mathrm{M})$ was incubated with the DMC1 presynaptic filament assembled on either the homologous ssDNA (panel I) or heterologous ssDNA (panel II) and the protection of the dsDNA against digest by Ssp1 was assessed (lanes 6 and 7). Various controls (lanes 2-5) were included. DNA protection was contingent upon ATP being present (lane 8). The results were plotted. Error bars represent \pm SD calculated based on at least three independent experiments.

Fig. 3. Lack of physical or functional interaction of RAD51AP1 Isoform 3 with DMC1.

(A) Affinity pulldown analyses using GST-tagged RAD51AP1 (I) and MBP-tagged RAD51AP1 (II) isoforms revealed that Isoform 3 does not interact with DMC1 (I, lane 9). Control pulldown reactions using GST and MBP were also included. The supernatant $(\mathrm{S})$, wash $(\mathrm{W})$, and SDS eluate (E) fractions of the pulldown reactions were analyzed by SDS-PAGE.

(B) While RAD51AP1 Isoform 1 (lanes 8 and 9) and Isoform 2 (lanes 4 and 5) were equally capable of enhancing the DMC1-mediated D-loop reaction in an ATP-dependent manner (lanes 6 and 10), RAD51AP1 Isoform 3 (lanes 12 and 13) was devoid of such ability. The amount of each RAD51AP1 Isoform used was 400 or $600 \mathrm{nM}$. The percentages of D-loop products formed were quantified and plotted.

(C) Isoform 1 and Isoform 2 were both found capable of functional synergy with DMC1 in duplex DNA capture (lanes 4 and 7) in an ATP-dependent fashion (lanes 5 and 8), 
Isoform 3 was much less effective (lane 10). Various controls were included. The amount of each RAD51AP1 Isoform used was $600 \mathrm{nM}$. The results were plotted.

(D) While RAD51AP1 Isoform 1 and Isoform 2 were both capable of functional synergy with DMC1 in synaptic complex assembly (lanes 5, 6, 9 and 10) in an ATP-dependent fashion (lanes 7 and 11), Isoform 3 was much less effective (lanes 13 and 14). Various controls were included. The amount of MBP-tagged RAD51AP1 Isoform 1 and GSTtagged RAD51AP1 Isoform 2 or Isoform 3 used was 300 or $500 \mathrm{nM}$. The results were plotted.

$(B-D)$ Error bars represent the standard deviation $( \pm \mathrm{SD})$ calculated based on at least three independent experiments.

Fig. 4. RAD51AP1 co-localizes with DMC1 foci on meiotic chromatin.

(A) Example of a spermatocyte nucleus in leptonema. Chromosome spreads were stained with anti-SYCP3 (blue), anti-DMC1 (red), and anti-RAD51AP1 GP49 (green) antibodies. Scale bar $=10 \mu \mathrm{m}$.

$(B-D)$ Zoomed-in displays of regions indicated in $A$ that harbored co-localized foci of RAD51AP1 and DMC1. Note that foci co-localization was scored without restriction to the subset of foci that share the same centroid.

$(E, F)$ Rotation experiment evaluating the statistical significance of RAD51AP1-DMC1 co-localization. $E$ is a zoomed-in display of the $20 \times 20 \mu \mathrm{m}$ box marked in $A$ within which co-localized RAD51AP1 and DMC1 foci were counted, as indicated by white arrows. $F$ corresponds to the same region in $E$ but with the RAD51AP1 immunofluorescence channel rotated $180^{\circ}$. RAD51AP1 foci that overlap with DMC1 foci after image rotation, as indicated by white arrowheads, are considered fortuitously colocalized.

$(G)$ Counts of axis-associated DMC1, RAD51AP1, and co-localized foci in leptotene stage spermatocytes. Each dot represents the count from one nucleus and the same 16 nuclei were quantified (bars $=$ mean \pm SD).

(H) Co-localized foci as a percentage of DMC1 or RAD51AP1 total foci. Each dot represents the percentage from one nucleus and 16 nuclei were quantified (bars = mean \pm SD).

(I) Results of a rotation experiment wherein axis-associated DMC1 or RAD51AP1 foci were counted before and after $180^{\circ}$ rotation of the DMC1 or RAD51AP1 immunofluorescence channel. Each dot represents the count from one nucleus and 8 nuclei were quantified (bars $=$ mean \pm SD).

$(J)$ Co-localized foci as a percentage of DMC1 total foci before and after $180^{\circ}$ rotation of the RAD51AP1 immunofluorescence channel. Each dot represents the count from one nucleus and 8 nuclei were quantified $($ bars $=$ mean \pm SD).

Competing interests statement: The authors declare that they have no competing financial interests 


\section{A}

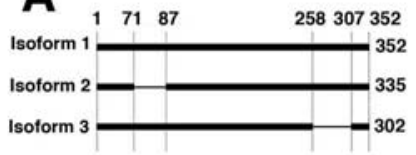

B

(I)

Clarified Extract<smiles>[I-][Tl]</smiles>

SP Sepharose

$\downarrow$

Amylose resin

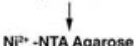

$\mathrm{Ni}^{2 *}$-NTA Agarose

$\downarrow$

Mono Q
(II) Clarified Extract

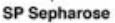

Glutathione resin

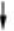

Source Q

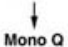

D

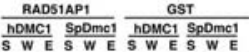

kDa

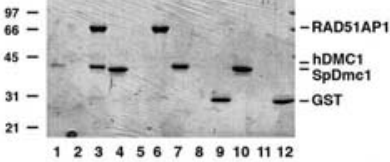

트

(I)

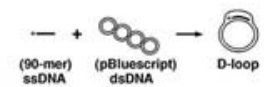

(II)
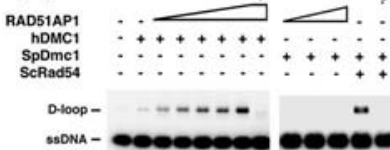

$\begin{array}{lllllllllllll}1 & 2 & 3 & 4 & 5 & 6 & 7 & 8 & 9 & 10 & 11 & 12 & 13\end{array}$

(III)

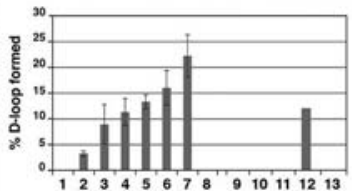

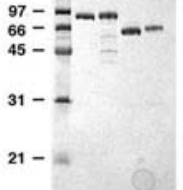




\section{Figure 2}

A

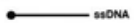

res once

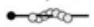

f RADSIAPI
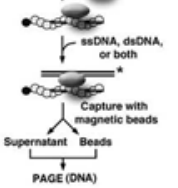

D
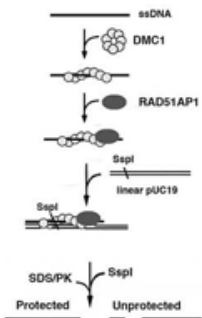

B

(I)

荤

RADS1AP1
DMC1

Supernatant $\quad-\infty-\infty-\infty-d$ DNA

(II)

$$
-0 \text { dsDNA }
$$

$\begin{array}{lllllll}1 & 2 & 3 & 4 & 5 & 6 & 7\end{array}$

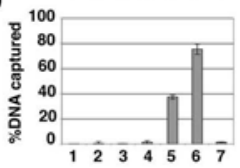

$\frac{2}{8}$

E

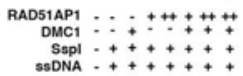

(I)

Homologous ssDNA

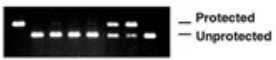

(II)

Heterologous ss DNA

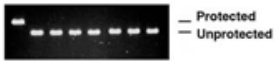

(iii)
(I)

RADS1AP1
DMC1
ssDNA ++++++
dsDNA ++++++




\section{Figure 3}

A

(i)

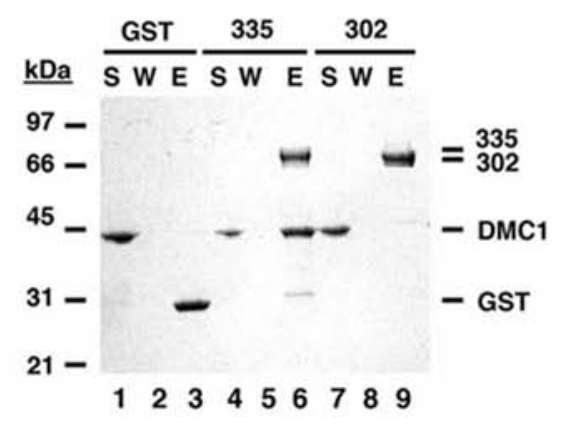

(ii)

kDa $\frac{335}{S W E} \frac{352}{S W E} \frac{M B P}{S W E}$

$97 \rightarrow-$
$66 \rightarrow-$

$45--- \pm-=-\mathrm{DMC1}_{\mathrm{DBP}}$

$31-$

$21-$

1223045567789

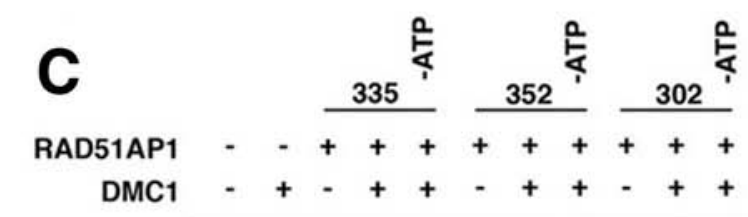

Supernatant

- - - dsDNA

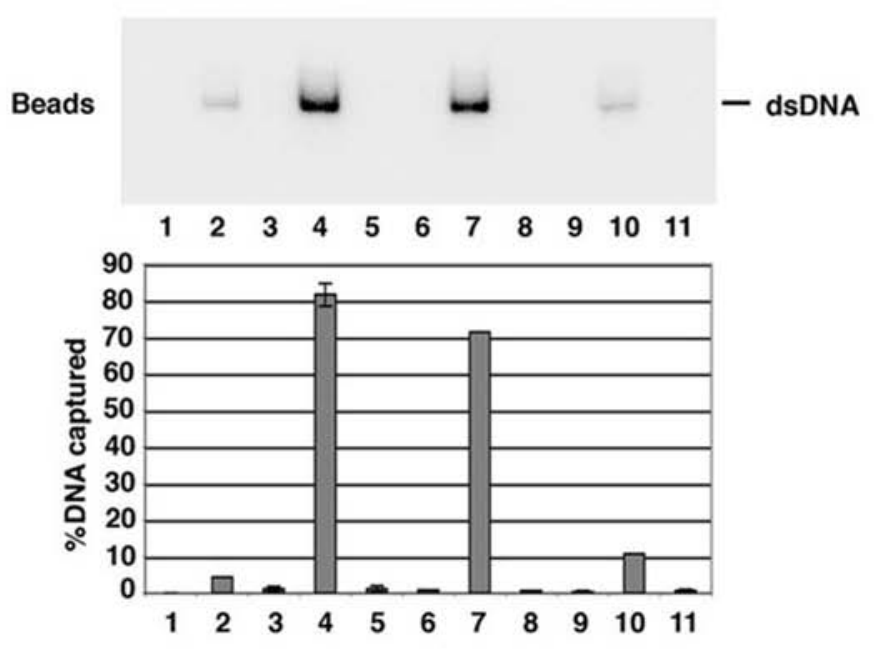

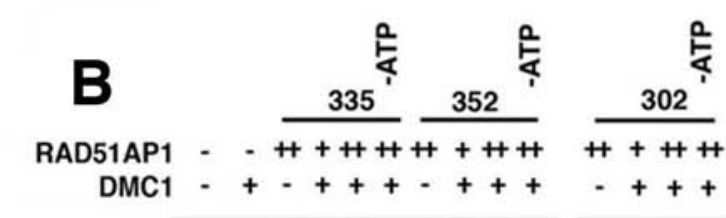
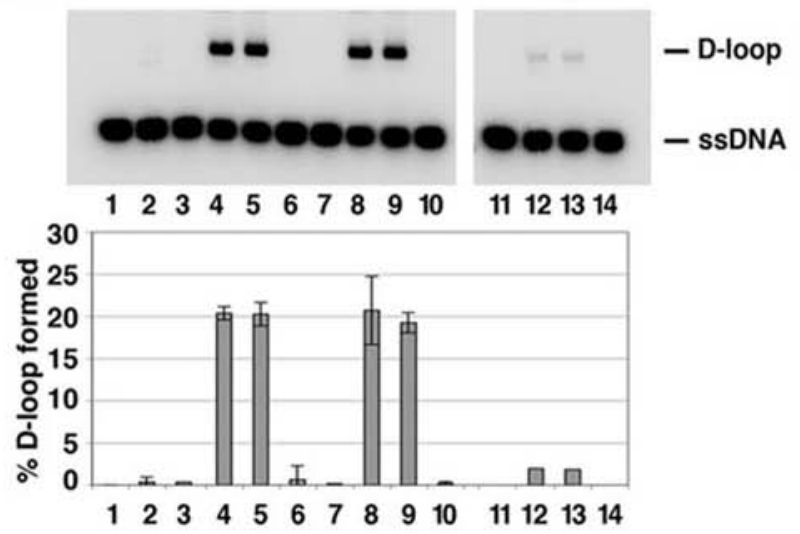

D

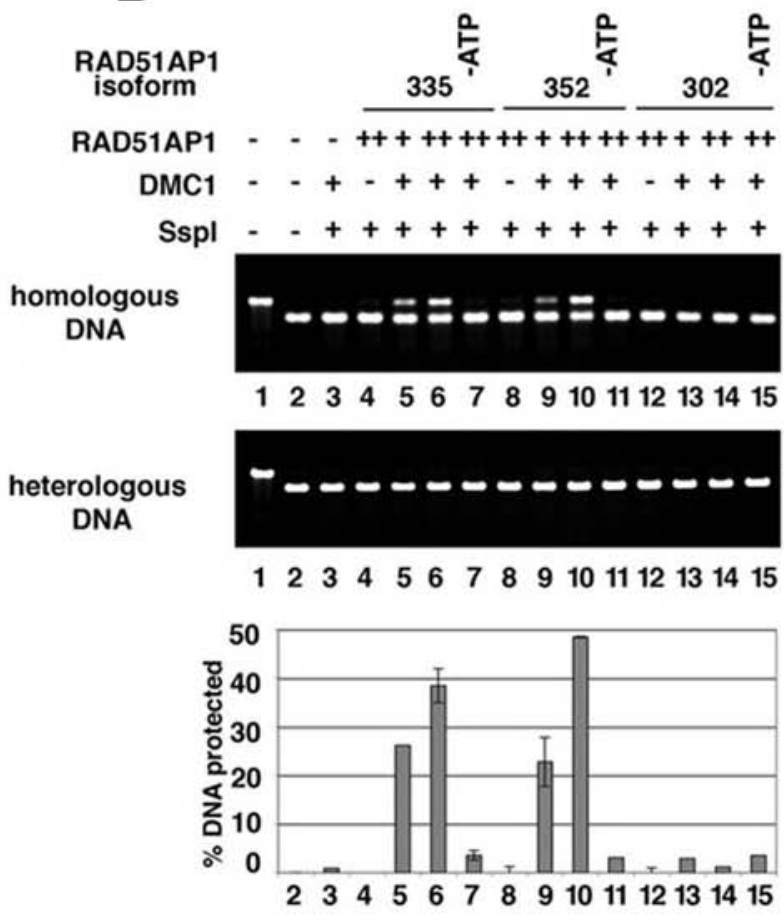




\section{Figure 4}

\section{a}

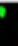

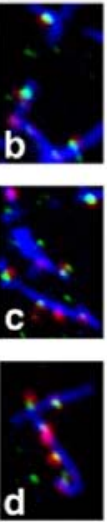

i
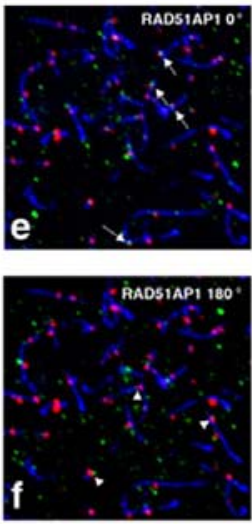

j g

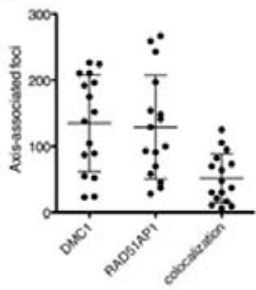

h

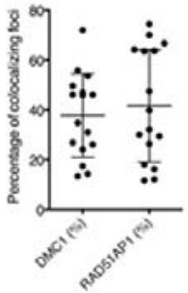

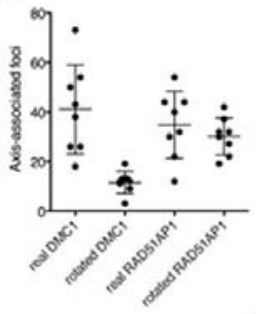

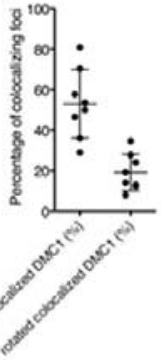




\section{SUPPORTING INFORMATION}

Cytological Analyses of Protein Foci. As expected, DMC1 and RAD51 foci were nearly exclusively axis-associated (co-localizing with SYCP3) and appeared throughout the nucleus in leptonema and zygonema, but were restricted to the unpaired X chromosome in early pachynema and completely disappeared by late pachynema (Fig. S7, $A-D$ ). RAD51AP1 staining patterns were qualitatively different, but with some features in common with DMC1 staining. We observed five classes of RAD51AP1 nuclear staining patterns that appeared in a stage-specific manner. Class I foci are axis-associated, and Class II foci are nuclear but not axis-associated (co-localizing only with DAPI) (Fig. S7A inset). Both classes were present throughout prophase I (Fig. S7, $A-D$ ). Class III staining represents the substantial coating of synapsed regions that begins to appear in zygonema (Fig. S7B inset) and culminates in pachynema (Fig. S7C inset), when all the bivalents are fully synapsed. In particular, much of the Class III AP1 staining was coincident with the persistent DMC1 and RAD51 foci that are associated with the unsynapsed portion of the $\mathrm{X}$ (and to a lesser extent the Y) during pachytene. However, since Class III staining was also observed in the non-homologously synapsed regions at the zygonema-like stage in chromosome spreads from Spo11-/- males where no meiotic DSBs are formed (Fig. S7F inset), we can infer this immunostaining pattern as being HR-independent and therefore unlikely to be informative about interactions of RAD51AP1 with DMC1. Class IV foci are large speckles that appeared both inside and outside of nuclei; when in the nucleus, they were rarely axis-associated (Fig. S7C inset). Since these foci occurred in all meiotic stages and were not diminished by treatment of the anti-RAD51AP1 antibodies with purified RAD51AP1 protein, we interpret them as non-specific background signal. Finally, Class V represents a small number of bright axis-associated foci (typically 1-2) that were observed on most late pachytene bivalents (Fig. S7D inset). This is consistent with a role of RAD51AP1 at later stages of meiosis, but likely independent of its interactions with DMC1 and RAD51.

Analysis of RAD51AP1-RAD51 Co-localization. The cytological analysis to examine the co-localization of RAD51 with RAD51AP1 was conducted as described in the main text for DMC1-RAD51AP1 co-localization test, using 1:200 rabbit anti-RAD51 antibodies (Calbiochem, PC130). We performed rotation tests to verify the significance of the RAD51-RAD51AP1 co-localization results (Fig. S8, E-F). We first rotated the RAD51 image and observed that the number of axis-associated foci decreased from $64 \pm$ 17 in the true images to $14 \pm 5$ in the rotated images (Fig. S8I, $P=0.0004$ ). The spreads with the rotated RAD51AP1 channel displayed an average of $65 \pm 16$ axis-associated RAD51AP1 foci, as compared to the $84 \pm 18$ observed in the unrotated nuclei (Fig. S8I, $P=0.0131)$. Importantly, before and after rotation of the RAD51AP1 image, the number of RAD51 foci co-localizing with RAD51AP1 decreased from $30 \pm 9$ to $7 \pm 2(P=$ $0.0004)$.

\section{Supporting Materials and Methods}


Purification of the Human HOP2-MND1 Complex. The E. coli (His)6-HOP2-MND1 expression plasmid was a kind gift from Shigeyuki Yokoyama (1), and we added a FLAG-tag to the carboxyl terminus of the MND1 gene in the plasmid before using it. An overnight culture $(250 \mathrm{ml})$ that harbored the aforementioned HOP2-MND1 co-expression plasmid was diluted in $10 \mathrm{~L}$ fresh LB broth and incubated at $37^{\circ} \mathrm{C}$ until the OD600 reached 0.6, at which time isopropyl 1-thio-D-galactopyranoside was added to $0.2 \mathrm{mM}$. After a $4 \mathrm{~h}$-incubation at $37^{\circ} \mathrm{C}$, cells were harvested by centrifugation and stored frozen at $-80^{\circ} \mathrm{C}$. All the subsequent steps were carried out at 0 to $4^{\circ} \mathrm{C}$. The cell pellet $(25 \mathrm{~g})$ was thawed in $30 \mathrm{ml}$ of cell breakage buffer $(50 \mathrm{mM}$ Tris- $\mathrm{HCl}, \mathrm{pH} 7.5,150 \mathrm{mM} \mathrm{KCl}, 2 \mathrm{mM}$ DTT, $10 \%$ sucrose, $10 \mathrm{mM}$ EDTA, and the following protease inhibitors: aprotinin, chymostatin, leupeptin, and pepstatin A at $3 \mu \mathrm{g} / \mathrm{ml}$ each) containing $0.01 \%$ Igepal (Sigma), and crude lysate was prepared by sonication. After centrifugation $(100,000 \mathrm{x} g$ for $90 \mathrm{~min}$ ), the clarified lysate was diluted with two volumes of buffer $\mathrm{T}$ ( $25 \mathrm{mM}$ Tris-

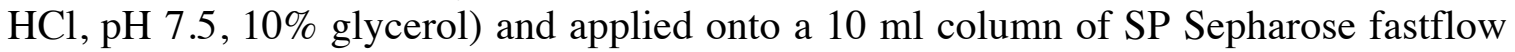
resin (Amersham Biosciences) pre-equilibrated with buffer $\mathrm{K}\left(20 \mathrm{mM} \mathrm{K \textrm {K } _ { 2 }} \mathrm{PO}_{4}, \mathrm{pH}\right.$ 7.4, $0.5 \mathrm{mMEDTA}, 1 \mathrm{mM}$ DTT, $10 \%$ glycerol, and $0.01 \%$ Igepal (Sigma)) containing $50 \mathrm{mM}$ $\mathrm{KCl}$, and developed with a $300 \mathrm{ml}, 50-450 \mathrm{mM} \mathrm{KCl}$ gradient. HOP2-MND1 eluted from the SP column at $\sim 200 \mathrm{mM} \mathrm{KCl}$, and peak fractions were pooled and incubated with $3 \mathrm{ml}$ of nickel nitrilotriacetic acid-agarose (Qiagen) for $2 \mathrm{~h}$. The affinity matrix was washed with $30 \mathrm{ml}$ of $20 \mathrm{mM}$ imidazole in buffer K containing $300 \mathrm{mM} \mathrm{KCl}$ before eluting the bound proteins with $10 \mathrm{ml}$ of $200 \mathrm{mM}$ imidazole in buffer T containing $50 \mathrm{mM} \mathrm{KCl}$. The eluate was diluted $10 \mathrm{ml}$ buffer $\mathrm{T}$ and fractionated in a $1 \mathrm{ml}$ Mono $\mathrm{S}$ column with a $15 \mathrm{ml}$ gradient of 50-400 $\mathrm{mM} \mathrm{KCl}$. Fractions containing the peak of nearly homogeneous HOP2-MND1 complex ( 1.2 mg eluting at $\sim 150 \mathrm{mM} \mathrm{KCl})$ were pooled and concentrated in an Amicon-30 micro-concentrator (Millipore) to $10 \mathrm{mg} / \mathrm{ml}$ and stored in small portions at $-80^{\circ} \mathrm{C}$.

Purification of Other Proteins. The L319Q and H329A mutant variants of MBP-tagged RAD51AP1 Isoform 2 were expressed in E. coli and purified as described for the wild type counterpart (Fig. 1B). Human RAD51 was purified as described previously (2).

Affinity Pulldown Experiments. The affinity pulldown assay involving tagged RAD51AP1 isoforms and RAD51 was performed as described (3). To examine whether RAD51AP1 and HOP2-MND1 interact (Fig. S10B), these protein species $(2 \mu \mathrm{M}$ each) were incubated in $30 \mu 1$ of buffer A (50 mM Tris at pH 7.5, $1 \mathrm{mM}$ DTT, $0.01 \%$ Igepal CA-630, $60 \mathrm{mM} \mathrm{KCl}$ ) for $30 \mathrm{~min}$ at $4^{\circ} \mathrm{C}$ before the reaction was mixed for $30 \mathrm{~min}$ at $4^{\circ} \mathrm{C}$ with $8 \mu 1$ of anti-FLAG M2 agarose resin (Sigma) to immobilize the HOP2-MND1 complex through the FLAG tag on MND1. After washing the beads twice with $200 \mu 1$ of the same buffer, bound proteins were eluted with $30 \mu 1$ of $2 \%$ SDS. The supernatant (S), wash (W), and SDS eluate (E), $8 \mu 1$ each, were analyzed by $12 \%$ SDS-PAGE and Coomassie Blue staining.

To ask whether HOP2-MND1 would interfere with RAD51AP1-DMC1 complex formation (Fig. S10D), DMC1 (3 $\mu \mathrm{M})$ was incubated with MBP-tagged RAD51AP1 (2 $\mu \mathrm{M})$ and increasing concentrations of HOP2-MND1 (3.4, 6.8 or $10.2 \mu \mathrm{M}$ of the complex) 
as above. The reaction was mixed with $8 \mu 1$ of Amylose-Sepharose resin (New England Biolabs) to immobilize RAD51AP1 via the MPB tag, followed by analysis as above.

Determination of Antibody Specificity. In order to determine the specificity of the antibodies used in the cytological analyses, we incubated the anti-RAD51, anti-DMC1, and anti-RAD51AP1 antibodies $(2.5 \mu \mathrm{g})$ with their cognate protein antigen $(12.5 \mu \mathrm{g}$ $\mathrm{RAD} 51 \mathrm{AP} 1$ or $6.4 \mu \mathrm{g}$ of either RAD51 or DMC1) for $30 \mathrm{~min}$ in $100 \mu \mathrm{l}$ before use. In the case of the anti-RAD51 and anti-DMC1 antibodies, the treatment specifically abolished the ability of the antibody preparations to reveal foci of their cognate target (Fig. S9D-E). In the case of the anti-RAD51AP1 antibodies, we found that all classes of RAD51AP1 foci disappeared subsequent to such treatment except the Class IV foci that we consider to be non-specific (Fig. S9A-B). Since the purified proteins used to titrate out the antibodies had not undergone any denaturation treatment, we infer that the antibodies used recognize the native form of their cognate protein antigen. As expected, the preimmune RAD51AP1 serum did not produce any protein foci (Fig. S9C).

D-loop Assay. The enhancement of the DMC1-mediated D-loop reaction by the indicated concentrations of RAD51AP1 (Isoform 2) and/or HOP2-MND1 was examined as described (3).

DNA Substrates. The following oligonucleotides (Integrated DNA Technologies) were used in the DNA mobility shift assay: P1 and P2 for ssDNA and dsDNA, D1, D2 and D3 to prepare the synthetic D-loop structure (See Table S2). DNA was labeled, annealed and purified as previously described (3).

DNA Mobility Shift Assay. The sequences of the oligonucleotides (Integrated DNA Technologies) used in substrate construction are listed in Table S2. Labeling of DNA and the DNA mobility shift assay with the ssDNA, dsDNA, and D-loop substrates (30 nM each) were carried out as described in our published study (3).

RT-PCR. Juvenile mice were sacrificed at 5, 15, and 21 days post partum (dpp) to obtain testis-specific RNA with an RNeasy Mini kit (Qiagen). Spo11-/- mice at $21 \mathrm{dpp}$ were also sacrificed to obtain testis-specific RNA with the same method. The SuperScript One-Step RT-PCR kit (Invitrogen) was used to synthesize cDNA and to amplify the RAD51AP1 transcript. Four primers were used to detect overlapping regions of the mouse RAD51AP1 transcript (RT1 to RT4, see Table S2 and Figure S6A). RT-PCR products were analyzed in a 1\% agarose gel run in TAE-buffer (40 mM Tris acetate, $\mathrm{pH} 7.4,2 \mathrm{mM}$ EDTA) and stained with ethidium bromide. DMC1 and $\beta$-actin primers were included as positive controls. Each primer pair set also included a minus (-) reverse transcriptase reaction to ensure that there was no genomic DNA contamination.

Western Blotting. Whole cell lysates were prepared from mouse testes (with tunica albuginea removed) by sonication in lysis buffer $(60 \mathrm{mM}$ Tris- $\mathrm{HCl}, \mathrm{pH} 6.8,2 \%$ SDS, $10 \%$ glycerol, $50 \mathrm{mM}$ dithiothreitol) and resolved by $10 \%$ SDS-PAGE. Proteins were transferred to a nitrocellulose membrane for Western analyses. For RAD51AP1 detection, the nitrocellulose blot was probed with the polyclonal guinea-pig antibody 
GP49, and then with goat anti-guinea pig IgG-HRP (sc-2438, Santa Cruz) as the secondary antibody. For DMC1 and $\beta$-actin detection, goat polyclonal anti-DMC1 antibody (sc-8973, Santa Cruz) or mouse monoclonal anti- $\beta$-actin antibody (A5316, Sigma) was used as the primary antibody, and either donkey anti-goat IgG-HRP (sc2020, Santa Cruz) or goat anti-mouse IgG-HRP (sc-2005, Santa Cruz) was used as the secondary antibody.

Immunofluorescence. The immunolocalization of RAD51AP1 with RAD51 was conducted as described in the main text, except that 1:200 rabbit anti-RAD51 antibody (Calbiochem, PC130) was used to detect RAD51.

\section{SUPPORTING REFERENCES}

1. Enomoto R, et al. (2006) Stimulation of DNA strand exchange by the human TBPIP/Hop2-Mnd1 complex. J Biol Chem 281:5575-5581.

2. Sigurdsson S, Trujillo K, Song B, Stratton S, Sung P (2001) Basis for avid homologous DNA strand exchange by human Rad51 and RPA. J Biol Chem 276:87988806.

3. Wiese C, et al. (2007) Promotion of homologous recombination and genomic stability by RAD51AP1 via RAD51 recombinase enhancement. Mol Cell 28:482-490.

\section{SUPPORTING FIGURE LEGENDS}

Fig. S1. GST-tagged RAD51AP1 Isoform 2 functionally synergizes with the stabilized DMC1 presynaptic filament.

(A) The D-loop reaction was conducted with RAD51AP1 (200, 300, and $500 \mathrm{nM})$ and 2 $\mathrm{mM} \mathrm{CaCl}$ to stabilize the DMC1 presynaptic filament. The results were plotted.

(B) Panel (I) shows that duplex DNA capture did not occur if ssDNA was omitted from the magnetic beads (lanes 1 and 2); a positive control (lane 3) was included. The amount of RAD51AP1 was 300 or $600 \mathrm{nM}$. Panel (II) shows that DMC1 and RAD51AP1 were retained on the magnetic beads when ssDNA was present (+DNA; lanes 3 and 4) but not when the DNA was omitted (-DNA; lanes 1 and 2). The beads were treated with SDS and the eluate fraction (designated as $E$ ) was analyzed along with the supernatant (designated as $\mathrm{S})$.

(C) Duplex capture was examined with RAD51AP1 (300 nM) in the presence of $2 \mathrm{mM}$ $\mathrm{CaCl}_{2}$. The results were plotted.

Fig. S2. Lack of functional synergy of GST-tagged RAD51AP1 Isoform 2 with SpDmc1.

Results showing that RAD51AP1 did not synergize with SpDmc1 in duplex capture $(A)$ or synaptic complex assembly $(B)$. The amount of RAD51AP1 was 300 or $600 \mathrm{nM}$ in $(A)$ and $500 \mathrm{nM}$ in $(B)$.

Fig. S3. Physical and functional interactions of the RAD51AP1 isoforms with RAD51.

(A) Results from affinity pulldown experiments showing that all three isoforms of RAD51AP1 are capable of RAD51 interaction. 
(B) Results from D-loop reactions showing that all three isoforms of RAD51AP1 are proficient in enhancing the recombinase activity of RAD51. The amount of each RAD51AP1 isoform used was 300 or $500 \mathrm{nM}$.

Fig. S4. DNA binding properties of the RAD51AP1 isoforms.

(A) Schematic of the DNA substrates used. The asterisk indicates the oligonucleotide that was radiolabeled.

(B) DNA mobility shift assay with the indicated concentrations of the three RAD51AP1 isoforms and D-loop as a substrate. Lanes 4, 7, and 10 are SDS and proteinase K treatment of the DNA binding reaction, showing that there is no nuclease contamination in the purified proteins.

(C) DNA mobility shift assay with the indicated concentrations of the three RAD51 AP1 isoforms and a mixture of the D-loop, dsDNA, and ssDNA as substrate.

Fig. S5. Interactions of the MBP-tagged RAD51AP1 L319Q and H329A Isoform 2 mutants with DMC1.

(A) The RAD51AP1 L319Q and H329A mutant proteins (i) were purified and analyzed by SDS-PAGE (ii).

(B) Affinity pulldown assays showing that both the RAD51AP1 L319Q and H329A mutant proteins are capable of complex formation with DMC1.

$(C-E)$ Results showing that the RAD51AP1 L319Q and H329A mutants are capable of functional synergy with DMC1 in the D-loop reaction $(C)$, in duplex capture $(D)$, and synaptic complex formation $(E)$. The amount of RAD51AP1 or mutants used was 300 or $500 \mathrm{nM}$ in $(C)$ and $500 \mathrm{nM}$ in $(D)$ and $(E)$.

\section{Fig. S6. Meiotic expression of RAD51AP1.}

(A) Gene structures of human and mouse RAD51AP1, with their Uniprot accession numbers listed below. Human RAD51APl is composed of 10 exons, of which exons 4 and 9 are alternatively spliced to produce three isoforms. Isoform 1 contains all 10 exons, Isoform 2 lacks exon 4, and Isoform 3 lacks exon 9. Mouse RAD51AP1 lacks the exon 4 equivalent of the human counterpart but is otherwise organized identically (aligned by the dashed lines). We did not detect the splicing out of exon 8 (equivalent to human exon 9). Location of the RT-PCR primers used for detecting the mouse RAD51AP1 transcript are indicated by the arrows.

(B) RT-PCR on total RNA from testes of juvenile wild-type mice of the indicated ages and juvenile Spol1-/- mice. PCR without the reverse transcriptase (-RT) was conducted for total RNA from testes of juvenile wild-type mice at $21 \mathrm{dpp}$ as a negative control. $\mathrm{DMC} 1$ and $\beta$-actin were included as controls in the analysis.

$(C)$ Western blot analysis of testis extracts from juvenile wild-type mice (15 and 21 days post partum) using an antibody (GP49) generated against mouse RAD51AP1 protein, human DMC1, or $\beta$-actin. The asterisks denote cross-reacting bands.

Fig. S7. RAD51AP1 immunostaining pattern on meiotic chromatin.

Chromosome spreads were stained with anti-SYCP3 (blue), antibody generated against full-length mouse RAD51AP1 protein (green), and anti-DMC1 (red). Scale bar $=10 \mu \mathrm{m}$. 
$(A-D)$ are wild-type spreads, $(E-F)$ are Spo11-/- spreads. Example of a wild-type spermatocyte nucleus in $(A)$ leptonema (a zoomed-in display of the indicated region in the merged image is shown without the DMC1 channel to show Class I RAD51AP1 foci that are axis-associated and Class II RAD51AP1 foci that are not); (B) zygonema (a zoomed-in display of the indicated region in the merged image to show Class III RAD51AP1 foci that coat synapsed regions); $(C)$ early pachynema (zoomed-in displays of the two indicated regions in the merged image to show Class III RAD51AP1 foci that coat synapsed regions and Class IV RAD51AP1 foci that are deemed aggregated entities appearing inside and outside the nucleus; the DAPI channel was added to the Class IV zoomed-in display); (D) late pachynema (a zoomed-in display of the indicated region in the merged image to show Class V RAD51AP1 foci that are bright and axis-associated). Example of a Spo11-/- spermatocyte nucleus in $(E)$ leptonema (a zoomed-in display of the indicated region in the merged image to show Class I RAD51AP1 foci that are axisassociated and Class II RAD51AP1 foci that are not), and $(F)$ Zygonema-like (a zoomedin display of the indicated region in the merged image to show Class III RAD51AP1 foci that coat synapsed regions).

Fig. S8. Co-localization of RAD51AP1 with RAD51 on meiotic chromatin.

(A) Example of a spermatocyte nucleus in leptonema. Chromosome spreads were stained with anti-SYCP3 (blue), anti-RAD51 (red), and anti-RAD51AP1 GP49 (green) antibodies. Scale bar $=10 \mu \mathrm{m}$.

$(B-D)$ Zoomed-in displays of regions indicated in $A$ that harbored co-localized foci of RAD51AP1 and RAD51. Note that foci co-localization was scored without restriction to the subset of foci that share the same centroid.

$(E, F)$ Rotation experiment to evaluate the statistical significance of RAD51AP1-RAD51 co-localization. $E$ is a zoomed-in display of the $20 \times 20 \mu \mathrm{m}$ box marked in $A$ within which co-localized RAD51AP1 and RAD51 foci were counted, as indicated by white arrows. $F$ corresponds to the same region in $E$ but with a $180^{\circ}$ rotation of the RAD51AP1 immunofluorescence channel. Any RAD51AP1 foci that overlap with RAD51 foci after image rotation, as indicated by white arrowheads, are considered fortuitously colocalized.

$(G)$ Counts of axis-associated DMC1, RAD51AP1, and co-localized foci in leptotene stage spermatocytes. Each dot represents the count from one nucleus and the same 14 nuclei were quantified (bars $=$ mean $\pm \mathrm{SD}$ ).

(H) Co-localized foci as a percentage of RAD51 or RAD51AP1 total foci. Each dot represents the percentage from one nucleus and 14 nuclei were quantified (bars = mean \pm SD).

(I) Results of a rotation experiment wherein axis-associated RAD51 or RAD51AP1 foci were counted before and after $180^{\circ}$ rotation of the RAD51 or RAD51AP1 immunofluorescence channel. Each dot represents the count from one nucleus and 9 nuclei were quantified (bars $=$ mean $\pm \mathrm{SD}$ ).

$(J)$ Co-localized foci as a percentage of RAD51 total foci before and after $180^{\circ}$ rotation of the RAD51AP1 immunofluorescence channel. Each dot represents the count from one nucleus and 9 nuclei were quantified (bars $=$ mean $\pm \mathrm{SD})$. 
Fig. S9. RAD51AP1, DMC1, and RAD51 antibody specificity.

(A) Example of a spermatocyte nucleus in leptonema. Chromosome spreads were stained with anti-SYCP3 (red) and anti-RAD51AP1 GP49 (green) antibodies, then mounted in Vectashield with DAPI (4',5-diamino-2-phenylindole). Scale bar $=10 \mu \mathrm{m}$. Class I and Class II foci (as definted in S7) were no longer detected after incubation of the antibody preparation with RAD51AP1 protein. Class IV foci remained.

(B) Example of a spermatocyte nucleus in pachynema. Chromosome spreads were stained with anti-SYCP3 (red) and anti-RAD51AP1 GP49 (green) antibodies, then mounted with DAPI. Scale bar $=10 \mu \mathrm{m}$. Class III and Class V foci (as defined in S7) were no longer detected after incubation of the antibody preparation with RAD51AP1 protein. Class IV foci remained.

(C) Example of a spermatocyte nucleus in leptonema (top) or pachynema (bottom). Chromosome spreads were stained with anti-SYCP3 (red) antibody and preimmune GP49 serum (green), then mounted with DAPI. Scale bar $=10 \mu \mathrm{m}$.

(D) Example of a spermatocyte nucleus in zygonema. Chromosome spreads were stained with anti-SYCP3 (red) and anti-DMC1 (green) antibodies, then mounted with 4',5diamino-2-phenylindole. Scale bar $=10 \mu \mathrm{m}$. Axis-associated DMC1 foci were no longer detected after incubation of the antibody preparation with $\mathrm{DMC1}$ protein, but incubation with RAD51 protein had little or no effect.

(E) Example of a spermatocyte nucleus in zygonema. Chromosome spreads were stained with anti-SYCP3 (red) and anti-RAD51 (green) antibodies, then mounted with DAPI. Scale bar $=10 \mu \mathrm{m}$. Axis-associated RAD51 foci were no longer detected after incubation of the antibody preparation with RAD51 protein, but incubation with DMC1 protein had little or no effect. 
Table S1 Conservation of RAD51AP1 isoforms among vertebrates

\begin{tabular}{|c|c|c|c|}
\hline Organism & Isoform 1 & Isoform 2 & Isoform 3 \\
\hline Human & 352 & 335 & 302 \\
\hline Chimpanzee & 352 & 335 & 302 \\
\hline Monkey & 352 & 335 & 302 \\
\hline Gorilla & 352 & - & - \\
\hline Dog & 354 & - & - \\
\hline Rock Hyrax & 353 & - & - \\
\hline Bat & 349 & - & - \\
\hline Horse & - & 330 & - \\
\hline Cow & - & 329 & - \\
\hline Chicken & - & 346 & - \\
\hline Pig & - & 340 & - \\
\hline Rat & - & 337 & - \\
\hline Mouse & - & 337 & - \\
\hline Tarsier & - & 327 & - \\
\hline Frog & - & 331 & - \\
\hline
\end{tabular}

The various isoforms of RAD51AP1 were retrieved from ensembl.org and determined to be homologous to the human isoforms 1,2, or 3 by sequence alignment. Numbers refer to total number of amino acid residues, i.e. 352 for 352 residues.

Table S2 Oligonucleotides used in this study

\begin{tabular}{|c|c|c|c|}
\hline Name & Sequence & Nature & Assay \\
\hline P1 & $\begin{array}{l}\text { 5'TTATATCCTTTACTTTGAATTCTATGTTTAACCTTTTACTTATTTTGTATTAGCCGG } \\
\text { ATCCTTATTTCAATTATGTTCAT-3' }\end{array}$ & ssDNA & $\begin{array}{l}\text { Duplex capture } \\
\text { EMSA }\end{array}$ \\
\hline P2 & $\begin{array}{l}\text { 5'ATGAACATAATTGAAATAAGGATCCGGCTAATACAAAATAAGTAAAAGGTTAAA } \\
\text { CATAGAATTCAAAGTAAAGGATATAA-3' }\end{array}$ & dsDNA & $\begin{array}{l}\text { Duplex capture } \\
\text { EMSA }\end{array}$ \\
\hline $\begin{array}{l}\text { D2 } \\
\text { D3 }\end{array}$ & $\begin{array}{l}\text { 5'GCCGTGTCACTGGATCAGAGGTCACTTGGCAAGGATGGCCCGTCCGTAGCACCAG } \\
\text { GACACCCTAGTTAGCTCCGACATGTCGTACATATCGGATGCTGGC-3' } \\
\text { 5'GCCAGCATCCGATATGTACGACATGTCGGAAATTAAGATGCAGCTTTGAAACTAA } \\
\text { CCATTTCTACTGGATGCCAAGTGACCTCTGATCCAGTGACACGGC-3' } \\
\text { GGTGCTACGGACGGGCCATCCTT-3' }\end{array}$ & $\begin{array}{l}\text { D-loop } \\
\text { substrate }\end{array}$ & EMSA \\
\hline $\begin{array}{l}\text { SspI } \\
\text { AflIII }\end{array}$ & $\begin{array}{l}\text { AATGTTGAATACTCATACTCTTCCTTTTTCAATATTATTGAAGCATTTATCAGGGTTA } \\
\text { CAGAATCAGGGGATAACGCAGGAAAGAACATGTGAGCAAAAGGCCAGCAAAAGCC }\end{array}$ & $\begin{array}{l}\text { homologous } \\
\text { heterologous }\end{array}$ & Synaptic assay \\
\hline $\begin{array}{l}\text { RT1 } \\
\text { RT2 } \\
\text { RT3 } \\
\text { RT4 }\end{array}$ & $\begin{array}{l}\text { 5'-ATGGTGCGTCCTACCAGAAATAGAAAACCA-3' } \\
\text { 5'-TACTTGGTTGGTGAGTGTTGGAAGTTCCTT-3' } \\
\text { 5'- GTTGCCCTGGCTTTATCTGTGAAGGAAC-3 } \\
\text { 5'- CCGCACTTGGCTGCTTGTGG-3' }\end{array}$ & & RT PCR \\
\hline
\end{tabular}




\section{Fig. S1}

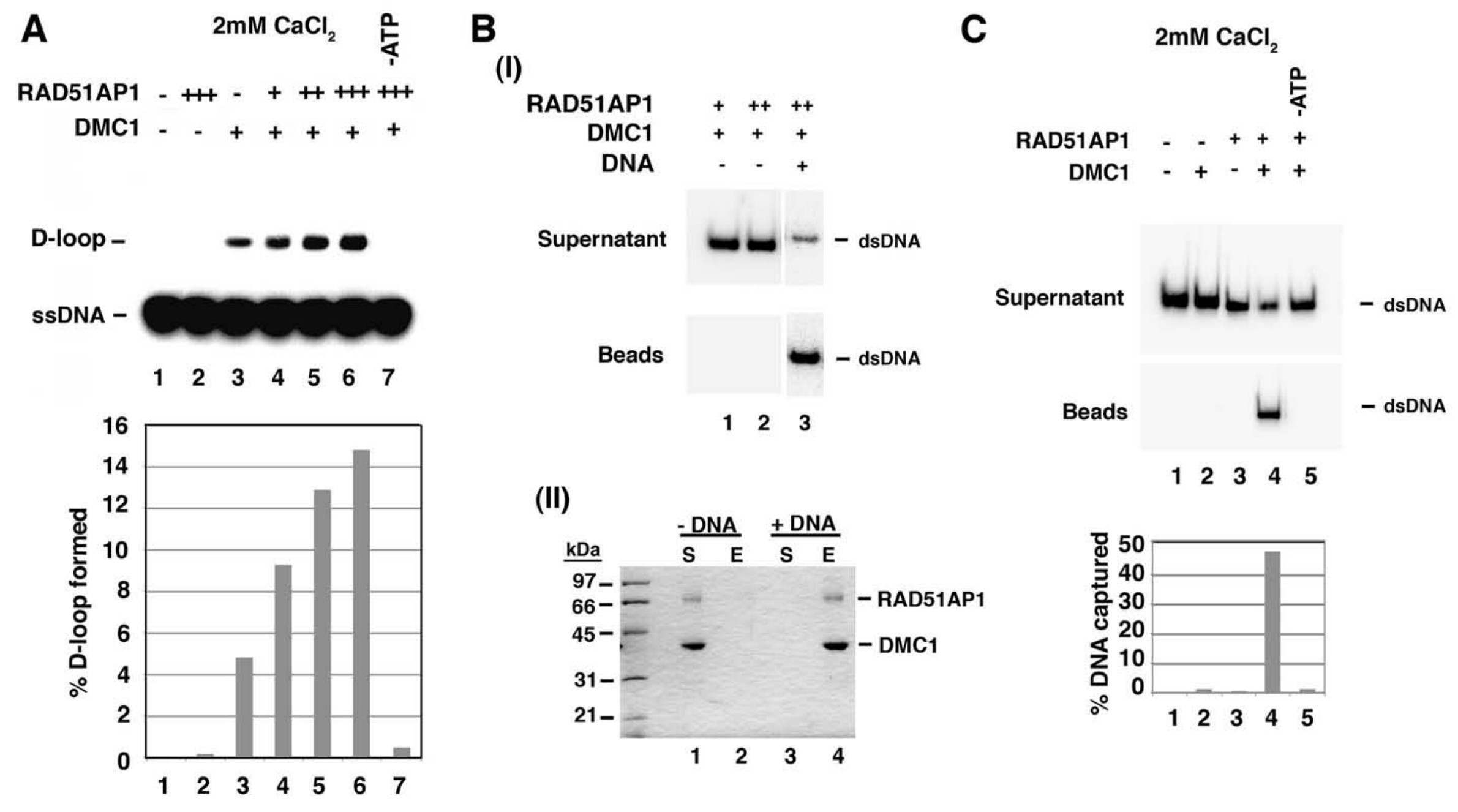




\section{Fig. S2}

A

$\begin{array}{rccccc}\text { RAD51AP1 } & - & ++ & + & ++ & ++ \\ \text { SpDmc1 } & - & - & + & + & - \\ \text { hDMC1 } & - & - & - & - & +\end{array}$

\section{Supernatant}

- dsDNA

— dsDNA

$\begin{array}{lllll}1 & 2 & 3 & 4 & 5\end{array}$

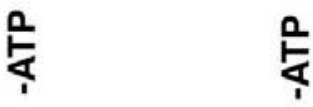

RAD51AP1 - $\quad-\quad-\quad+\quad+\quad-\quad+$ hDMC1 - $-\quad-\quad-\quad+\quad++$ SpDmc1 - $-++\quad+\quad-\quad-$ Sspl -+++++++

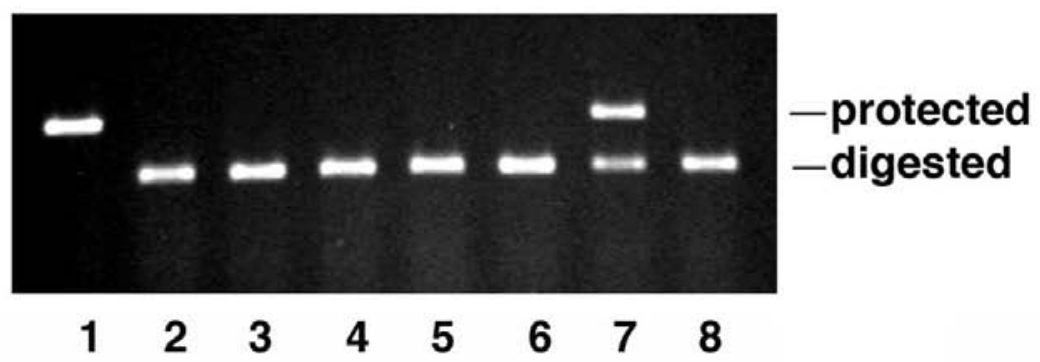




\section{Fig. S3}

Dray2010_FigS3

$\mathbf{A}$
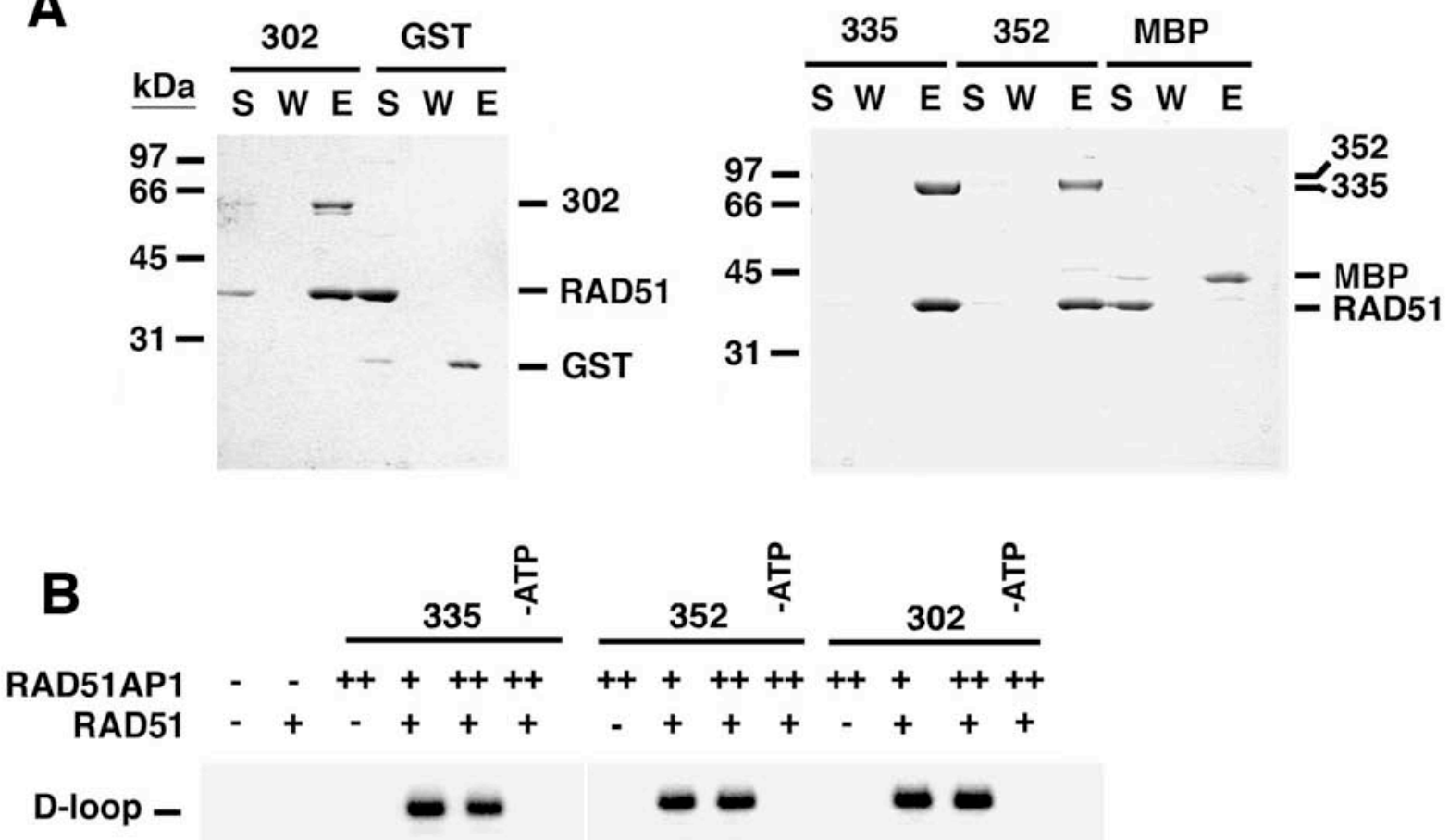

$\begin{array}{llllllllllllll}1 & 2 & 3 & 4 & 5 & 6 & 7 & 8 & 9 & 10 & 11 & 12 & 13 & 14\end{array}$

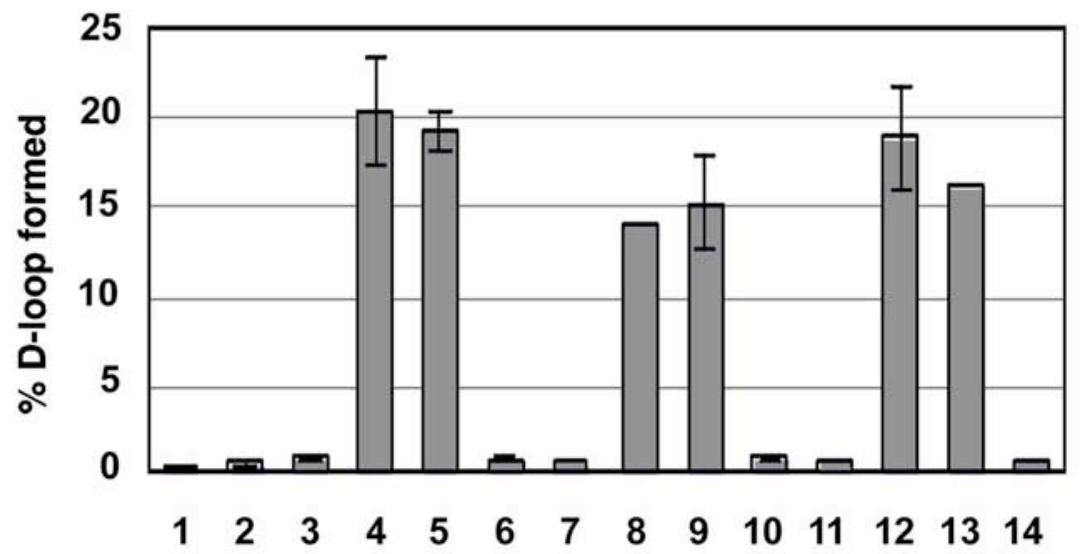




\section{Fig. S4}

A

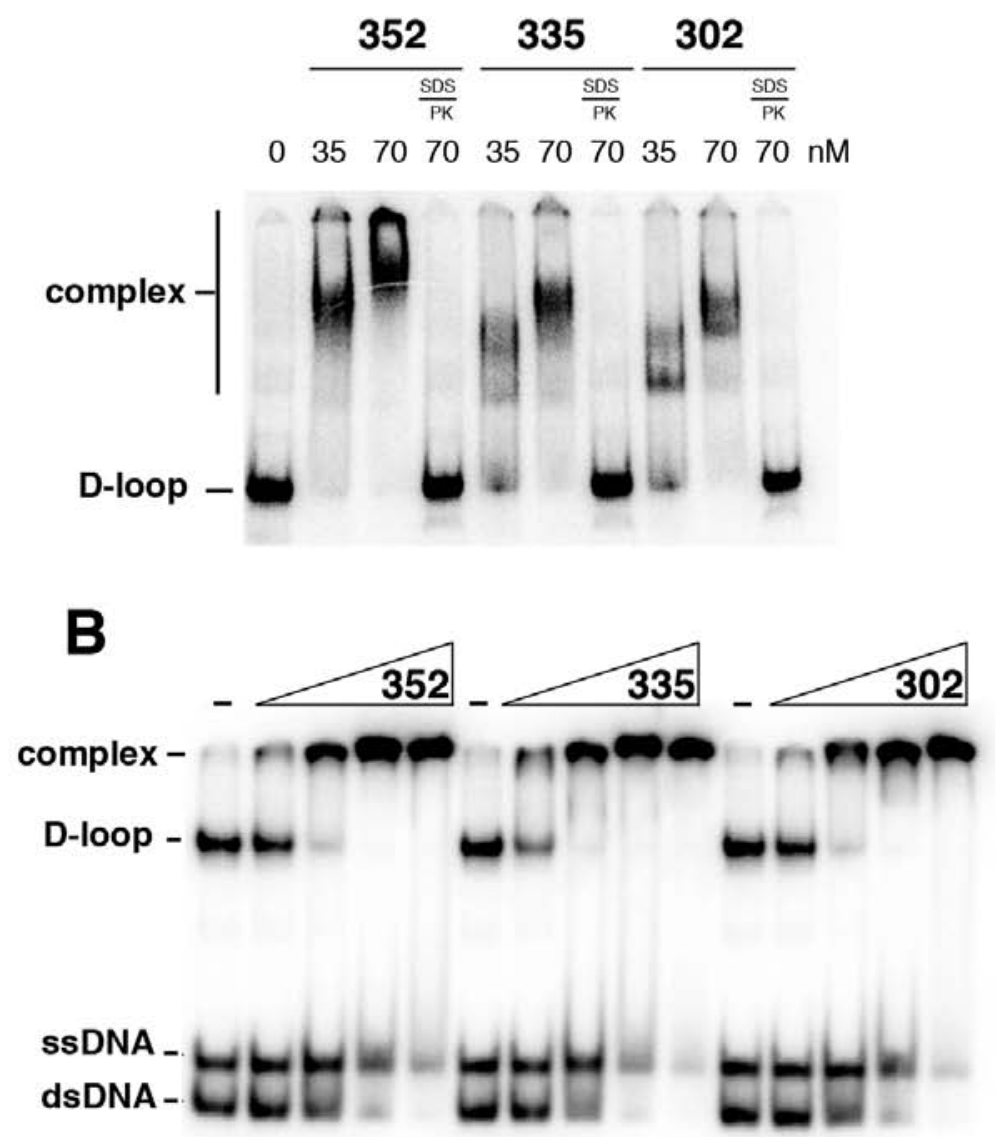

C

(i)

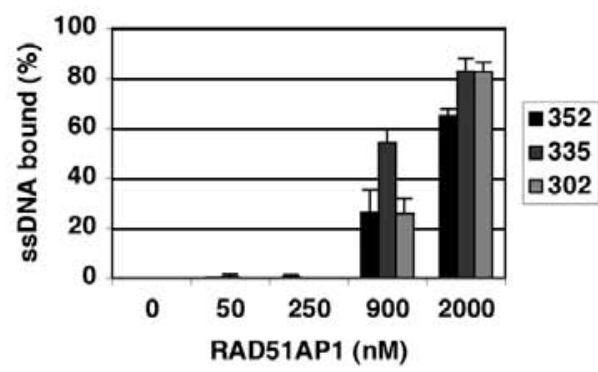

(ii)

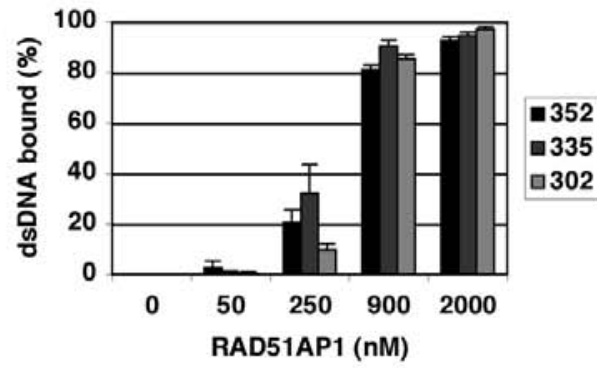

(iii)

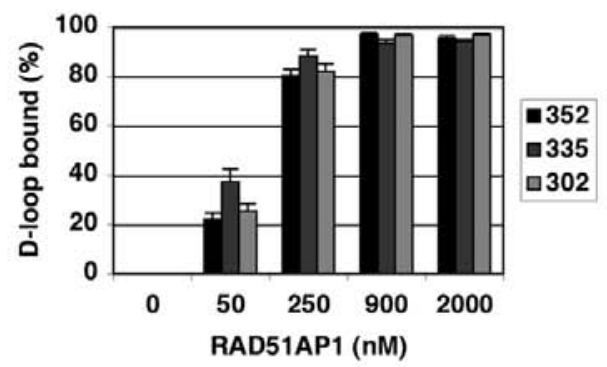




\section{Fig. S5}

A

(I)

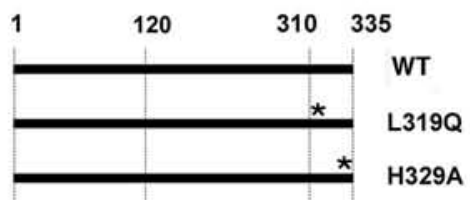

(II)

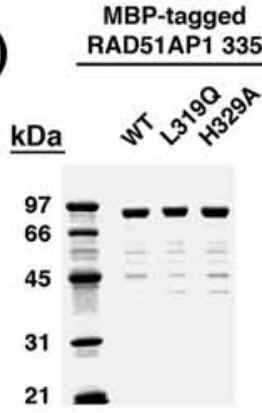

B

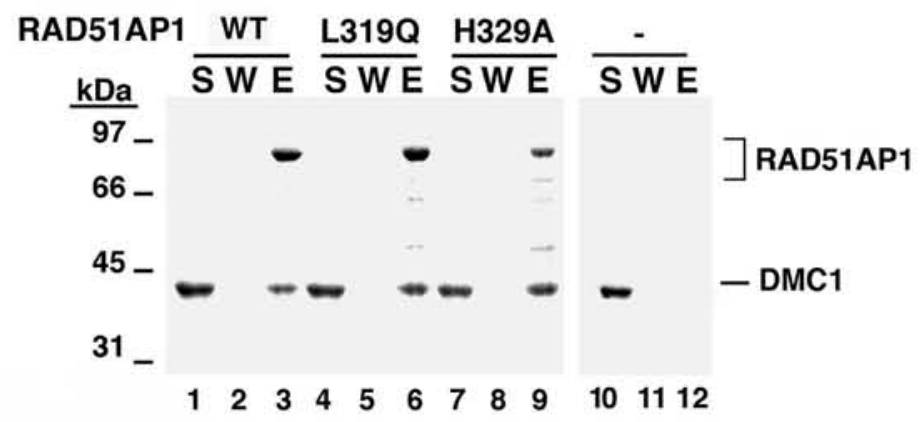

D

(I) WT L L319Q H329A

RAD51AP1

DMC1

D-loop ssDNA -

$$
\begin{array}{lllllllll}
1 & 2 & 3 & 4 & 5 & 6 & 7 & 8 & 9
\end{array}
$$

(II)

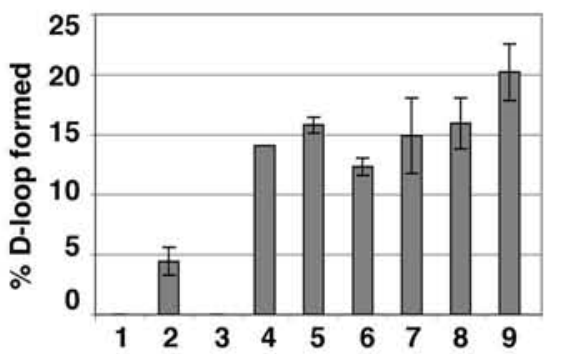

E

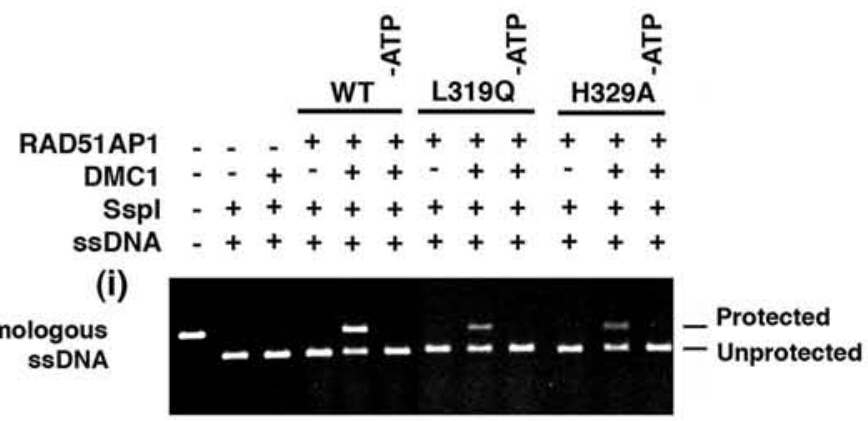

(ii)

Heterologous ss DNA

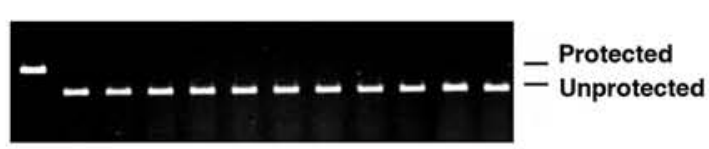

(iii) $\begin{array}{llllllllllll}1 & 2 & 3 & 4 & 5 & 6 & 7 & 8 & 9 & 10 & 11 & 12\end{array}$

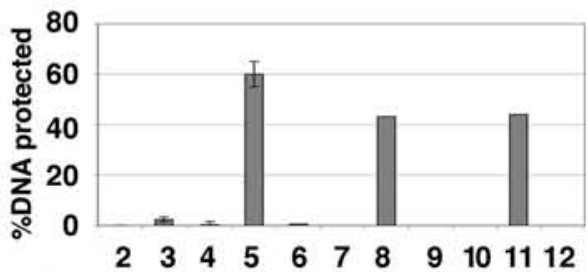

RAD51AP1 - - $\frac{\text { WT }}{+++} \frac{\text { L319Q }}{+++t+t+}$ DMC1 - + + + - + +++

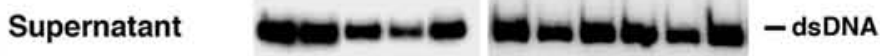

Beads

(II)

$\begin{array}{lllllllllll}1 & 2 & 3 & 4 & 5 & 6 & 7 & 8 & 9 & 10 & 11\end{array}$

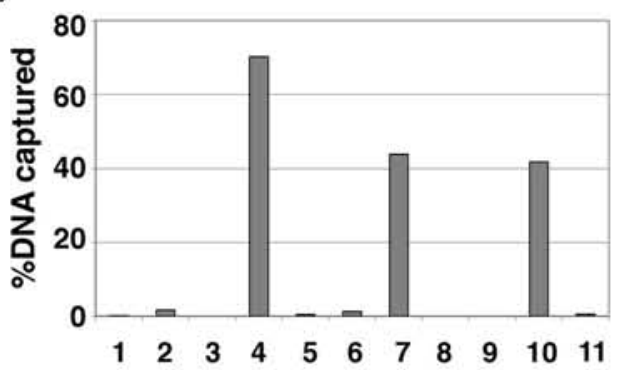




\section{Fig. S6}

A

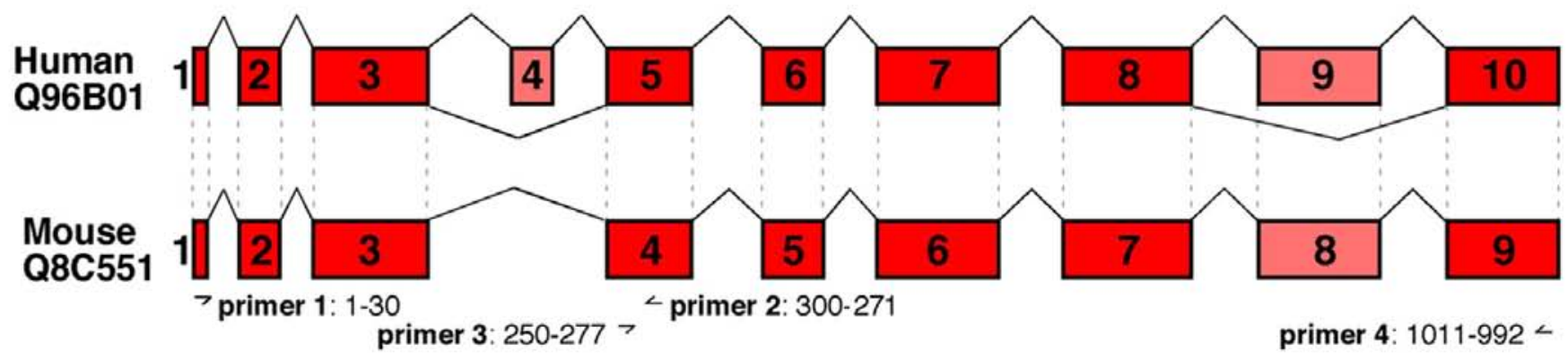

B

C
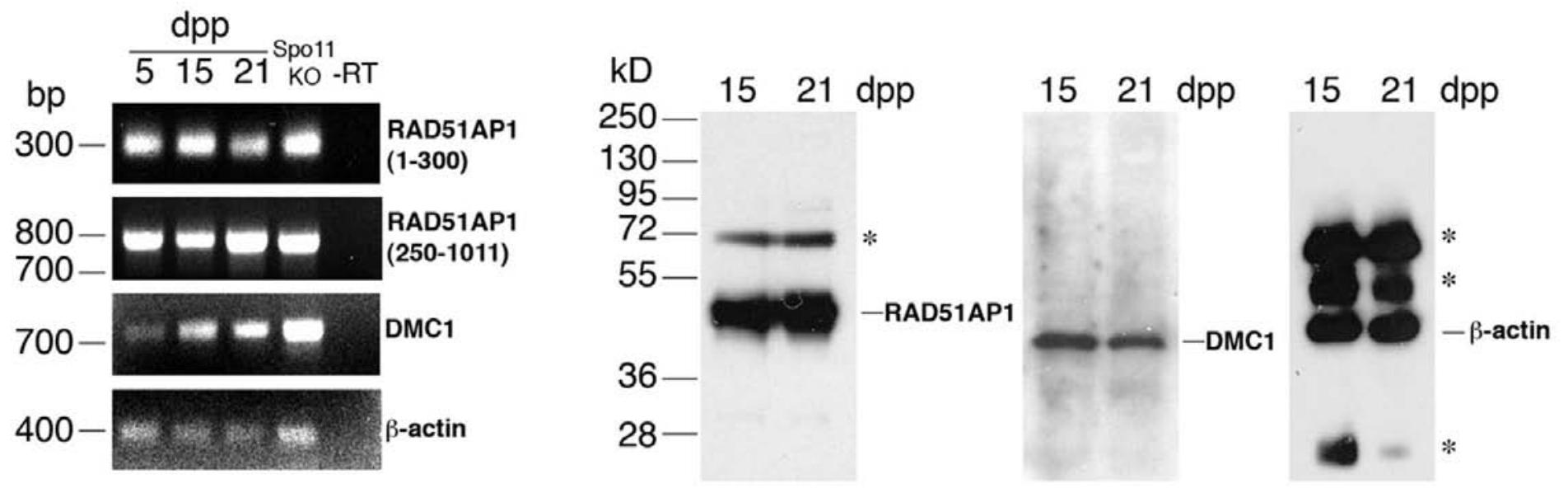


\section{Fig. S7}

A

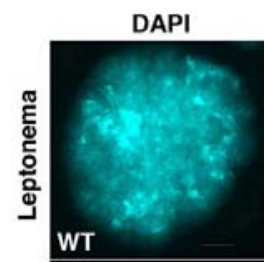

SYCP3

DMC1/RAD51AP1

merge

Class

B

C

D

졿
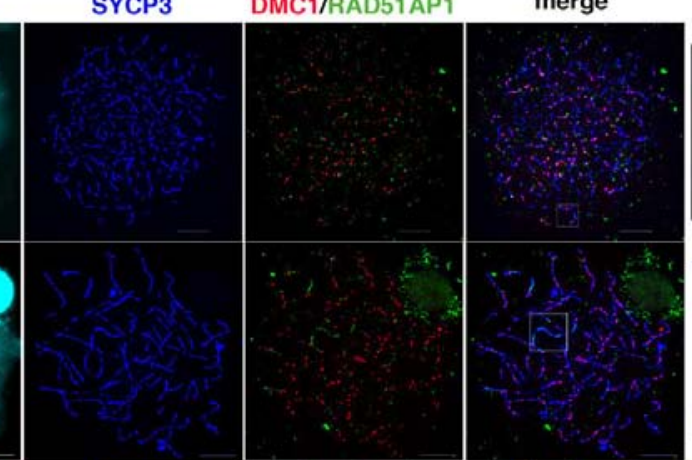

il

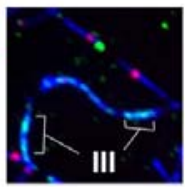

E

胥

WT 


\section{Fig. S8}

\section{A}
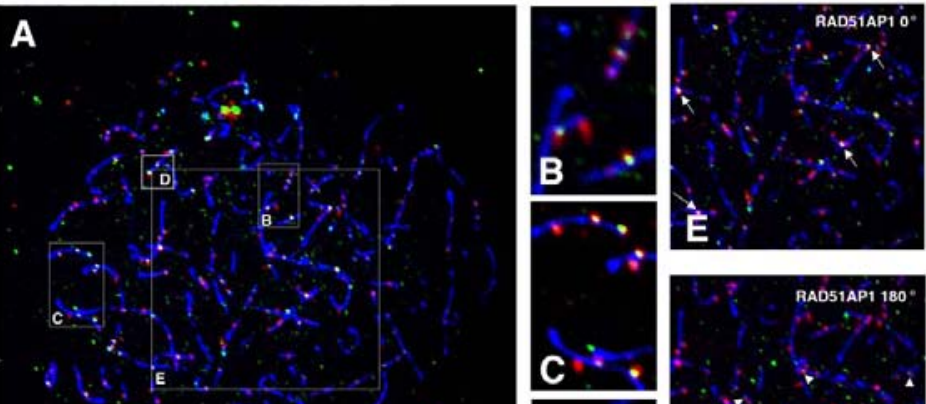

SYCPE

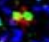

-

$$
\text { (1) }
$$

RAD51

RAD51AP1

G

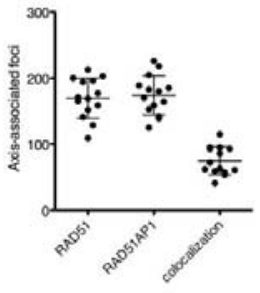

H

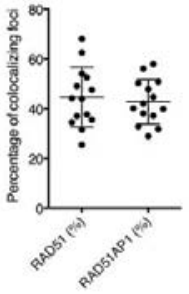

I

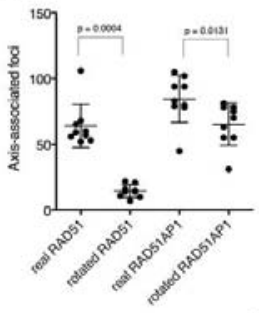

J

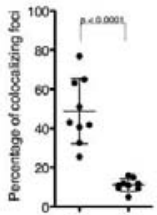

RAD51AP1 180

i

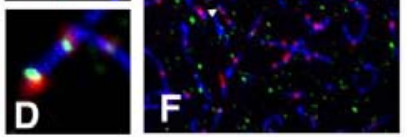




\section{Fig. $\mathbf{S 9}$}

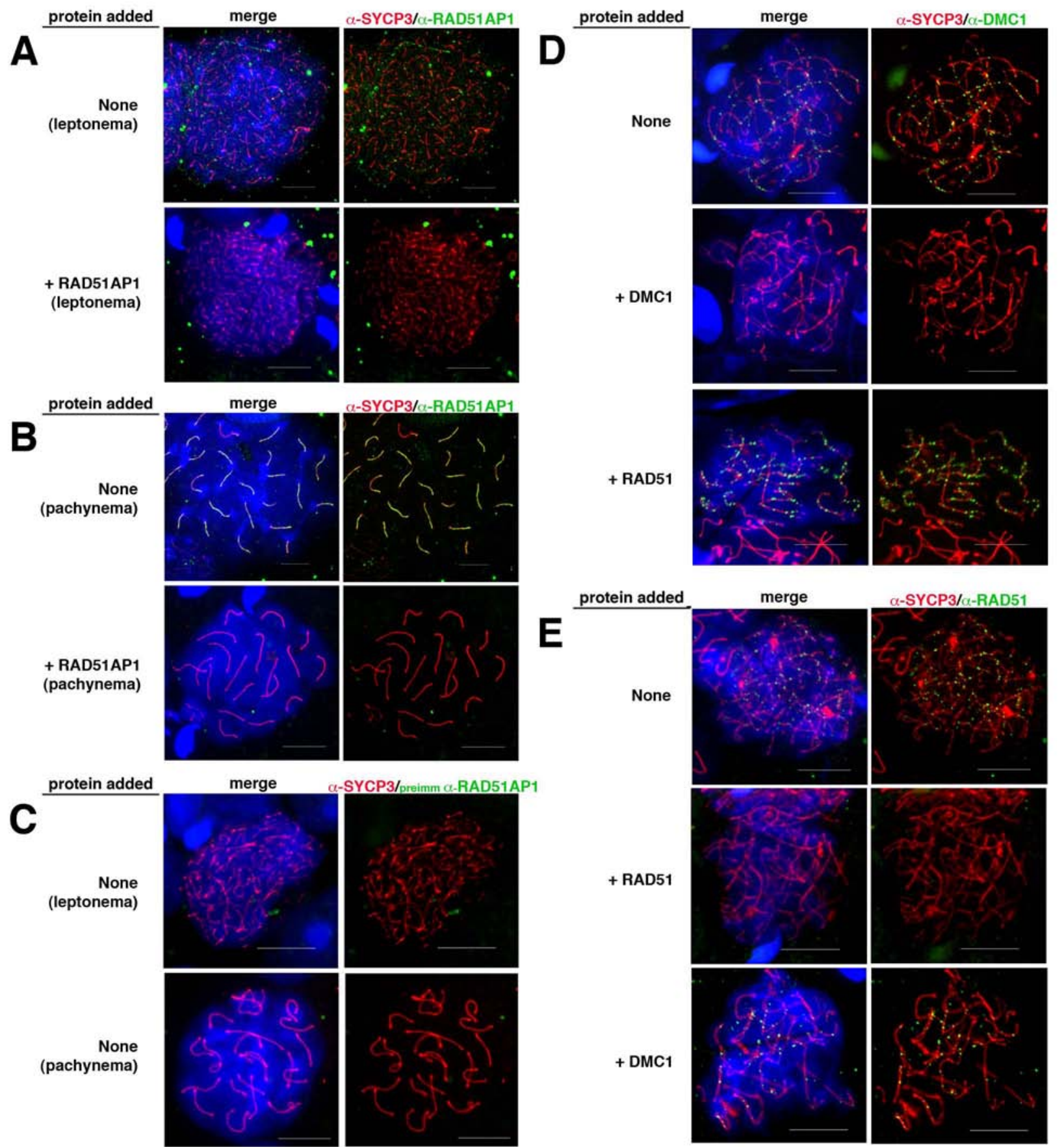

